\title{
Neural Network Based Techniques for Microwave Passive Components Modeling and Design
}

By

Xin Zhang, B. Eng.

\author{
A thesis submitted to \\ The Faculty of Graduate Studies and Research \\ in partial fulfilment of \\ the degree requirements of \\ Master of Applied Science \\ Ottawa-Carleton Institute for \\ Electrical and Computer Engineering
}

\author{
Department of Electronics \\ Carleton University \\ Ottawa, Ontario. Canada
}

August 2008

Copyright 0

2008 - Xin Zhang 


$\begin{array}{ll}\begin{array}{l}\text { Library and } \\ \text { Archives Canada }\end{array} & \begin{array}{l}\text { Bibliothèque et } \\ \text { Archives Canada }\end{array} \\ \begin{array}{l}\text { Published Heritage } \\ \text { Branch }\end{array} & \begin{array}{l}\text { Direction du } \\ \text { Patrimoine de l'édition }\end{array} \\ \begin{array}{l}\text { 395 Wellington Street } \\ \text { Ottawa ON K1A 0N4 } \\ \text { Canada }\end{array} & \begin{array}{l}\text { O95, rue Wellington } \\ \text { Ottawa ON K1A 0N4 } \\ \text { Canada }\end{array}\end{array}$

Your file Votre référence

ISBN: 978-0-494-44065-0

Our file Notre référence

ISBN: 978-0-494-44065-0

NOTICE:

The author has granted a nonexclusive license allowing Library and Archives Canada to reproduce, publish, archive, preserve, conserve, communicate to the public by telecommunication or on the Internet, loan, distribute and sell theses worldwide, for commercial or noncommercial purposes, in microform, paper, electronic and/or any other formats.

The author retains copyright ownership and moral rights in this thesis. Neither the thesis nor substantial extracts from it may be printed or otherwise reproduced without the author's permission.
AVIS:

L'auteur a accordé une licence non exclusive permettant à la Bibliothèque et Archives Canada de reproduire, publier, archiver, sauvegarder, conserver, transmettre au public par télécommunication ou par l'Internet, prêter, distribuer et vendre des thèses partout dans le monde, à des fins commerciales ou autres, sur support microforme, papier, électronique et/ou autres formats.

L'auteur conserve la propriété du droit d'auteur et des droits moraux qui protège cette thèse. $\mathrm{Ni}$ la thèse ni des extraits substantiels de celle-ci ne doivent être imprimés ou autrement reproduits sans son autorisation.
In compliance with the Canadian

Privacy Act some supporting forms may have been removed from this thesis.

While these forms may be included in the document page count, their removal does not represent any loss of content from the thesis.
Conformément à la loi canadienne sur la protection de la vie privée, quelques formulaires secondaires ont été enlevés de cette thèse.

Bien que ces formulaires aient inclus dans la pagination, il n'y aura aucun contenu manquant.

\section{Canada}




\section{Abstract}

This thesis presents the neural network based techniques for microwave passive components modeling and design. First, a modeling method called combined approach of transfer function and neural network (TF-NN) is described. Instead of using equivalent circuit, it uses transfer function to provide prior knowledge to the neural network such that the complexity of the neural network input-output mapping for the target problem is simplified. This reduction in mapping complexity helps to develop a neural network model with less training data while keeping the electromagnetic (EM) level accuracy. This TF-NN model is much faster than detailed EM simulation and is more flexible for general passive component modeling than equivalent circuit based knowledge model. Passive microwave components such as via and square inductor models are developed using the TF-NN method. Secondly, another new approach, which is aimed at designing one of the basic microwave components, microstrip power divider, is also proposed. The EM structure of the power divider is first segmented in order to simplify the structure. Detailed EM simulation time is reduced through the model segmentation. The neural networks are used to learn the EM behaviour of the power divider's sub structures. The trained neural sub models are then combined to form the overall power divider structure which can be used during model optimization and provide fast evaluation of the power divider's structure. The optimization time is significantly reduced by using the proposed design approach. 
Dedicated to:

My parents

\&

Jerry

For their endless love

- ii - 


\section{Acknowledgements}

It's a pleasure to take this opportunity to thank all the people who made this thesis come to reality.

The research work would not have come to fruition had it not been for the professional guidance of my supervisor Qi-Jun Zhang. Many thanks go to him for his words of advice and invaluable inspiration.

My special thanks to Dan Gorcea of Flextronics Canada (Ottawa) for providing motivation and technical inputs for the power divider simulation and optimization example.

My deepest thank is given to my most loyal supporter Jerry, who deserves a Masters in love for listening to my rants and dealing with my stress of finishing the thesis. There's simply no way I ever would finish without him. Many thanks also go to his parents, for their delicious dishes and smiles.

Finally, there is simply no amount of thanks that would be sufficient to express my appreciation for the endless love of my wonderful parents. Thank you for teaching me the most important thing of life is simply believing in yourself.

Again, thank you all for being a part of my journey and making it easier. 


\section{Table of Contents}

Abstract

Acknowledgements

Table of Contents iv

List of Tables $\quad$ vii

List of Figures $\quad$ viii

$\begin{array}{ll}\text { Chapter 1: Introduction } & 1\end{array}$

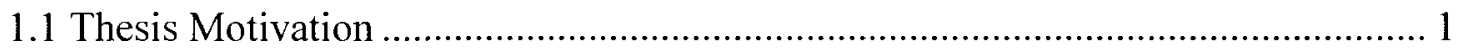

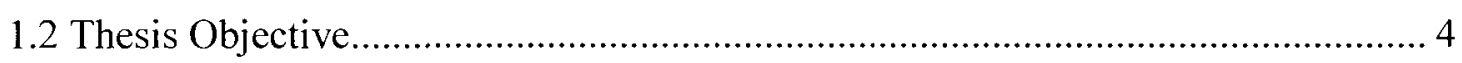

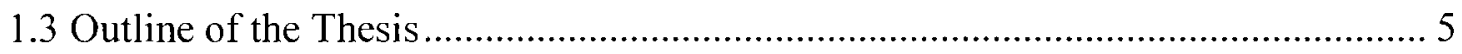

Chapter 2: Artificial Neural Networks (ANNs) for RF/Microwave Circuit Design: A

Review

2.1 Roles of ANNs in RF/Microwave Circuit Design .......................................... 7

2.2 Neural Network Model Development Overview ............................................ 9

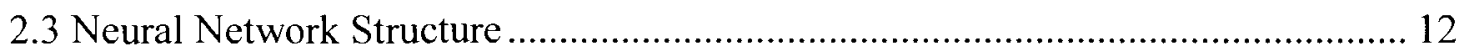

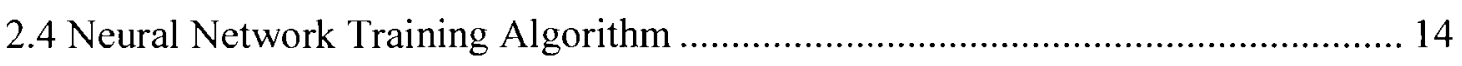

2.4.1 Back Propagation (BP) Training Algorithm ............................................. 16

2.4.2 Gradient-Based Optimization Techniques ................................................ 16

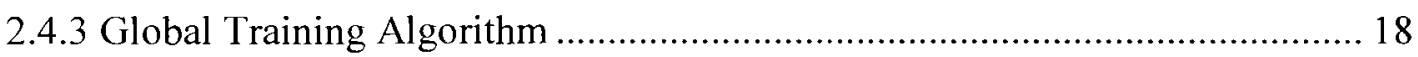

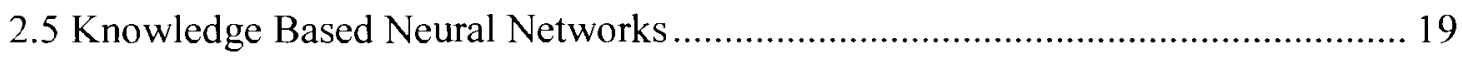

2.5.1 Knowledge-Based Neural Network (KBNN) …..................................... 20

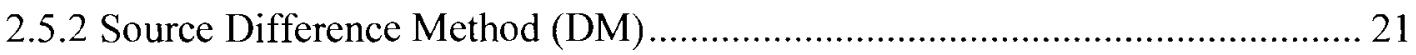

- iv - 
2.5.3 Prior Knowledge Input (PKI) Method ............................................................ 21

2.5.4 Space Mapping (SM) Method.................................................................... 22

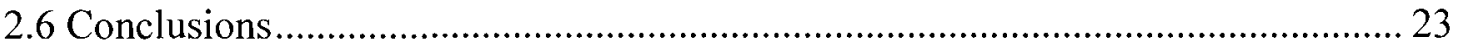

Chapter 3: A Combined Approach of the Transfer Function and the Neural Network (TF-NN) for Passive Microwave Components Modeling in Multilayer

Circuits $\quad 24$

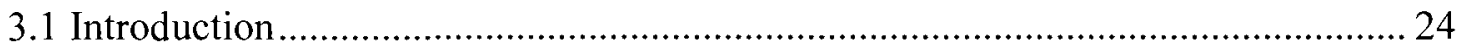

3.2 Combined Approach of Transfer Function and Neural Network (TF-NN) ........... 26

3.2.1 Neural Network (NN) Model: Problem Statement ........................................... 26

3.2.2 Combined Approach of Transfer Function and Neural Network (TF-NN)

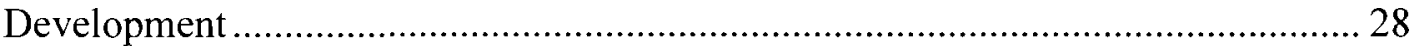

3.3 Application Examples for Passive Microwave Components Modeling Using the

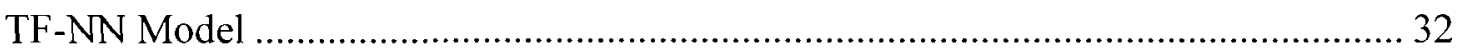

3.3.1 Via Model for Integrated Circuits.............................................................. 32

3.3.2 Via model for Printed Circuit Boards .............................................................. 35

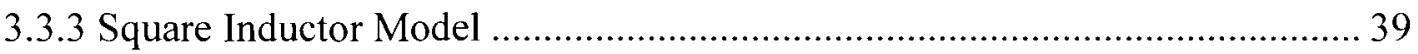

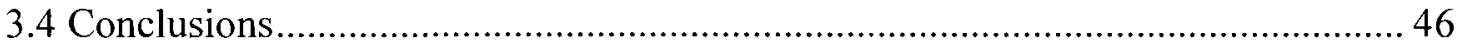

Chapter 4: A Neural Network Based Approach to the Design and Optimization of $\begin{array}{ll}\text { the Microstrip Power Divider } & 47\end{array}$

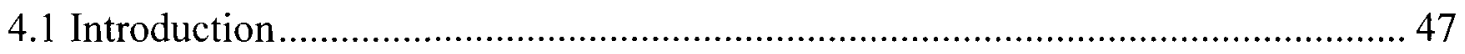

4.2 Proposed Design and Optimization Procedure ………………………………….... 49

4.2.1 Problem Statements ................................................................................... 49

4.2.2 Approximate Design Procedure ……………………………………....... 50

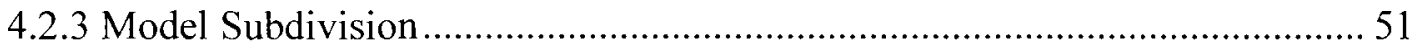

4.2.4 Neural Network (NN) Model Development ................................................. 52

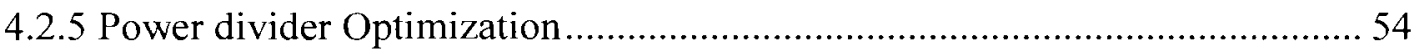

4.3 Demonstration Example of the Microstrip Power Divider Design and Optimization Using the Proposed Design Method ........................................................................ 55

4.3.1 EM Power Divider Structure Development ...................................................... 55 


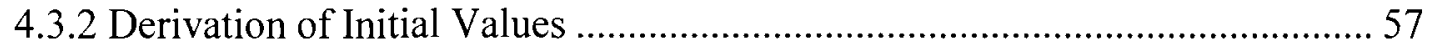

4.3.3 Power Divider Model Subdivision................................................................. 58

4.3.4 Neural Network (NN) Models Development and EM Data Generation.......... 62

4.3.5 Combined Neural Sub Model Development and Optimization Results.......... 66

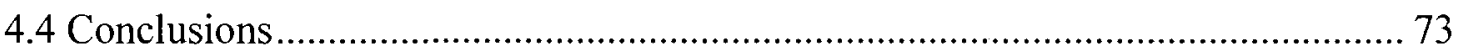

Chapter 5: Conclusion and Future Work $\quad 74$

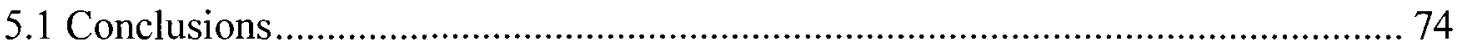

5.2 Suggestions for Future Research ................................................................... 75

$\begin{array}{ll}\text { Bibliography } & 77\end{array}$ 


\section{List of Tables}

Table 3-I. Data ranges of all the parameters used in developing the TF-NN model for the via in the integrated circuits. 33

Table 3-II. Training/test errors for the via model in the integrated circuits 35

Table 3-III. Data ranges of all the parameters used in developing the TF-NN model for the via in the multilayer circuit boards. 36

Table 3-IV. Training/test errors for the via model in the printed circuit boards. 37

Table 3-V. Data ranges of all the parameters used in developing TF-NN model for the square inductor. 40

Table 3-VI. Training/test errors of the square inductor model.

Table 3-VII. CPU time comparisons for all three examples by using the EM simulator and the TF-NN model.

Table 4-I. Training errors of the three neural sub models for the power divider design. . 64

Table 4-II. Training ranges of the three neural sub models of the power divider. 65

Table 4-III. Geometrical parameter values used in the power divider design and optimization example. 71

Table 4-IV. Comparisons of the CPU time for the power divider design and optimization using different simulation methods. 


\section{List of Figures}

Figure 2-1. Flowchart summarizing the procedure of developing a neural network model.

Figure 2-2. Multilayer perceptrons (MLP) structure (from Q. J. Zhang, K. C. Gupta and

V. K. Devabhaktuni [52]).

Figure 3-1. Structure of the TF-NN model: It is a hierarchical model containing two levels: The higher level is transfer function equations while the lower level is neural network model

Figure 3-2. MLP neural network structure used in the TF-NN model.

Figure 3-3. 3D structure of the via for integrated circuits. $D$ and $H$ are the design variables.

Figure 3-4. Test results of the via model for integrated circuits: (a): Test results are generated based on $D=0.29 \mathrm{~mm}, H=0.075 \mathrm{~mm}$. (b): Test results are generated based on $D=0.23 \mathrm{~mm}, H=0.105 \mathrm{~mm}$.

Figure 3-5. 3D structure of the via for printed circuit boards. $D$ and $H$ are the design variables. 36

Figure 3-6. Test results of the via model for printed circuit boards: (a): The test results are generated based on $D=0.55 \mathrm{~mm}, H=1.5 \mathrm{~mm}$. (b): The test results are

generated based on $D=0.77 \mathrm{~mm}, H=1.7 \mathrm{~mm}$. 38

Figure 3-7. Top view of the square inductor structure with adjustable parameters. 39

Figure 3-8. Comparisons of the real and imaginary part of the S11 for the square-spiral inductor using the TF-NN model and detailed EM simulation, Curve $\mathrm{A}$ and Curve $\mathrm{B}$ are generated based on $\mathrm{N}=1.5, R_{i n}=33 \mu \mathrm{m}, \mathrm{W}=6 \mu \mathrm{m}, \mathrm{S}=1.5 \mu \mathrm{m}$ and $\mathrm{N}=1.5, R_{\text {in }}=123 \mu \mathrm{m}, \mathrm{W}=6 \mu \mathrm{m}, \mathrm{S}=9.5 \mu \mathrm{m}$, respectively. The parameter values of the test structures $\mathrm{A}$ and $\mathrm{B}$ are never used in training. 
Figure 3-9. Comparisons of the real and imaginary parts of the $\mathrm{S} 12$ for the square-spiral inductor using the TF-NN model and detailed EM simulation, Curve A and Curve B are generated based on $\mathrm{N}=1.5, R_{i n}=33 \mu \mathrm{m}, \mathrm{W}=6 \mu \mathrm{m}, \mathrm{S}=1.5 \mu \mathrm{m}$ and $\mathrm{N}=1.5, R_{i n}=123 \mu \mathrm{m}, \mathrm{W}=6 \mu \mathrm{m}, \mathrm{S}=9.5 \mu \mathrm{m}$, respectively. The parameter values of the test structures A and B are never used in training. 43

Figure 3-10. Comparisons of the real and imaginary parts of the S22 for the square-spiral inductor using the TF-NN model and detailed EM simulation, Curve A and Curve $\mathrm{B}$ are generated based on $\mathrm{N}=1.5, R_{i n}=33 \mu \mathrm{m}, \mathrm{W}=6 \mu \mathrm{m}, \mathrm{S}=1.5 \mu \mathrm{m}$ and $\mathrm{N}=1.5, R_{i n}=123 \mu \mathrm{m}, \mathrm{W}=6 \mu \mathrm{m}, \mathrm{S}=9.5 \mu \mathrm{m}$, respectively. The parameter values of the test structures $A$ and $B$ are never used in training.

Figure 4-1. The power divider structure with adjustable parameters: W: Width of the rectangle, L: Length of the rectangle, S: Space between each pair of the coupled rectangles.

Figure 4-2. Illustration of the subdivision of the EM power divider structure................. 52

Figure 4-3. The MLP neural network structure used in power divider design................. 53

Figure 4-4. Rectangle power divider structure. ……...................................................... 56

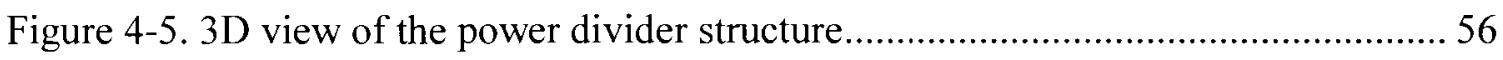

Figure 4-6. Equivalent circuit of the power divider built with Agilent-ADS................... 57

Figure 4-7. EM simulation results of the power divider structure based on initial optimized parameter values: $\mathrm{dB}$ S11, $\mathrm{dB}$ S12 and $\mathrm{dB}$ S13.

Figure 4-8. 14 sub structures of the overall power divider structure after model subdivision.

Figure 4-9. Four types of EM sub structures: (a) 1:2 power splitter, (b) Coupled difference between two rectangles, (c) Coupled lines, (d) Coupled output lines.

Figure 4-10. A comparison of the EM simulation results using the overall EM structure and the combined EM sub structure: The simulations are based on the power divider's initial parameter values. 
Figure 4-11. Three neural sub models used for the power divider design and optimization: (a) Neural sub model 1, (b) Neural sub model 2, (c) Neural sub model 3...6 63 Figure 4-12. Comparisons of simulation results using combined EM sub structure and combined neural sub model. 2 different power divider structures are used, which are listed in the third and fourth column of Table-III.......................6 67

Figure 4-13. Combined neural sub model for the power divider optimization. 68

Figure 4-14. A comparison of the optimized S1 1 and the initial S1 1 of the power divider.

Figure 4-15. The comparisons of the power divider simulation results computed using the combined neural sub model, the combined EM sub structure, the equivalent circuit and the overall EM structure. The structure parameter values for all these simulations are the optimized ones achieved by using the proposed method 


\section{Chapter 1:}

\section{Introduction}

\subsection{Thesis Motivation}

Over the past decades, with the help of Computer-Aided Design (CAD) tools, the circuit design in radio frequency (RF) and microwave areas has seen considerable growth. As the signal frequencies are getting higher, the circuit design becomes more complicated. As a result, more efficient CAD tools need to be developed to meet the advanced requirements in $\mathrm{RF}$ and microwave areas.

The process of CAD generally consists of three important aspects: modeling, analysis and optimization. Modeling has been recognized as the major difficulty in the successful implementation of the CAD techniques. Fortunately, the emerging of the electromagnetic (EM) simulation has brought a first-pass success to accurate modeling. However, when it comes to efficiency, EM computation by itself is too computationally intensive to be used in some CAD processes, e.g., statistical analysis and yield optimization, both of which usually take iterations to solve the complex circuits.

Another existing popular approach for components/circuits modeling is the analytical model, such as empirical model and equivalent circuit. However, many of these analytical models lack the detail and fidelity of their electrical behaviour. For example, in 
high frequency, the EM effects of a resistor can not be ignored. The existing equivalent model may have difficulty in reflecting these EM effects. Even if the EM effects are taken into account when creating equivalent circuit topology, it might still be difficult to get an accurate model when the interested parameters of the resistor are changed, or a new device is introduced.

In recent years, Artificial Neural Networks (ANNs) have emerged as a powerful technique for modeling general input-output relationships. ANNs have been widely used in various areas such as control [1], telecommunications [2], biomedicine [3], remote sensing [4], pattern recognition [5], manufacturing [6] and etc. In RF/microwave areas, ANNs have also been successfully applied to a variety of applications [7] such as automatic impedance matching [8], microstrip circuit design [9], microwave circuit analysis and optimization [10,11], active device modeling [12-15], passive components modeling [15-23], and modeling for electro/opto interconnections [24]. Neural network models can be easily developed through learning the electrical behaviour of passive/active components/circuits and subsequently used in high level circuit simulation and design, providing fast answers to the problems they have learned. One of the distinguished characteristics offered by ANNs is that ANNs can generalize, which means they can respond correctly to new data that have not been used for model development. Neural network can be more accurate than polynomial regression models [25-30], much faster than detailed EM simulation [31,39], and easier to develop when a new device/technology is introduced [30]. Neural network can handle more dimensions than 
look-up tables [30] and allow more automation in model development than conventional modeling approaches.

With continuing development in applications of the neural networks to microwave design, there is a growing need for cost reduction of the neural network model development. During the model development using the neural network, more data points in the model input parameter space are required to best represent the target problems when they become complicated. In other words, the quantity of the training data is decided by the complexity of the target problem. As a result, data generation can be expensive because of the need for a large amount of training data. In addition, the demands for new devices as well as more complex models are increasing as the wireless communication market keeps growing. Therefore, advanced requirements for ANNs approach are raised, e.g., to reduce the training data for developing neural network models while keeping a high level of model accuracy. The concept of knowledge based neural network [31] has been proposed to deal with this situation. The basic idea of the knowledge based neural networks is incorporating existing knowledge into the neural network model. The embedded existing knowledge can provide additional information for the neural network. Therefore, instead of learning from scratch, the neural network only needs to learn the information missed by the existing knowledge. The training data are significantly reduced by adding the prior knowledge. The extrapolation capability of the neural network is also improved by applying the knowledge-based concept. Four methods, which are knowledge-based neural network (KBNN) method [31], source difference method (DM) [18], prior knowledge input (PKI) method [32] and space 
mapping (SM) method [33], have been published recently along the direction of the knowledge-based concept. The details of these methods are reviewed in Section 2.5.

The prior knowledge used in the knowledge based neural network is mainly in the form of empirical model or equivalent circuit. If the empirical model or equivalent circuit of an RF/microwave component does not exist or has not been developed yet, no existing knowledge can be incorporated into the neural network. Therefore, another motivation of this thesis is to explore more efficient ways than embedding empirical/equivalent circuit into the neural network to shorten the design procedure but still keep a high level of accuracy.

\subsection{Thesis Objective}

As stated in this thesis's motivation, the main purpose of this thesis is to utilize neural network to make it more efficient to develop fast and accurate models. Two neural network based techniques are presented in this thesis:

1. A hierarchical modeling approach, called a combined model of transfer function and neural network (TF-NN), is described. This TF-NN model employs the knowledge-based concept and is aimed at reducing the mapping complexity of the existing neural network methods. By using this TF-NN method, the amount of training data is significantly reduced while the resulting model is sill accurate, and moreover, fast and accurate models can be achieved without exploring the equivalent circuit topology. Application examples, which are via model and square inductor model, will be developed using this method. 
2. A neural network approach to the design and optimization of the microstrip power divider is developed. Model subdivision is carried out first to simplify the power divider's EM structure. After that, neural networks are used to learn the EM behaviour of the sub structures of the power divider. Optimization of the power divider is based on these trained neural models instead of the detailed EM simulation. This proposed approach is proved to speed up the optimization procedure considerably compared to the conventional power divider design process.

\subsection{Outline of the Thesis}

This thesis is organized as follows:

In chapter 2, the role of ANNs and their applications in RF/microwave CAD are discussed at the beginning. The procedure of neural network model development is then briefly reviewed. Neural network structure and the training algorithm, which are the two major issues in developing neural network models [34], are described in details. Along the direction of knowledge based neural network design, four existing methods will be explicitly explained.

In chapter 3, a combined approach of transfer function and neural network (TF-NN) is discussed in details. The development process of the TF-NN model is presented in depth first. The advantage of the TF-NN model is demonstrated through three application examples, which are the via model for printed circuit boards, the via model for integrated circuits and the square inductor model. The CPU efficiency is also proved by comparing the TF-NN model outputs with the simulation results of the EM field solver. 
Chapter 4 introduces a novel neural network based approach for the microstrip power divider design and optimization. Instead of optimizing the detailed EM structure directly, the microstrip power divider is optimized based on neural network models which are built from learning the EM data of the power divider's sub structures. Model subdivision, neural network learning and model optimization are discussed in this chapter. A power divider design example using the proposed method is provided to demonstrate the efficiency and validity of this design approach.

Conclusions and suggestions for future research are discussed in Chapter 5. 


\section{Chapter 2:}

\section{Artificial Neural Networks (ANNs) for RF/Microwave}

\section{Circuit Design: A Review}

The neural network approach for $\mathrm{RF} /$ microwave circuit design has gained tremendous attention because of its advantage of being fast and flexible. The importance and details of the neural network techniques are further discussed in this chapter. Another important topic of combining existing knowledge with neural network is also reviewed here.

\subsection{Roles of ANNs in RF/Microwave Circuit Design}

Recently, wireless communication has progressed considerably and led to a burst of interests in improving the existing design methods in the RF and microwave areas. Meanwhile, the rapid development of the wireless communication industry starts to put emphasis on time-to-market and lower costs, which places enhanced demands on Computer-Aided Design (CAD) tools for RF/microwave circuit design. Therefore, effective CAD is essential to the success of the modern wireless communication industry.

During the CAD process, the most critical step is the accurate and efficient modeling of the RF and microwave components. It has been well-recognized [35] that the accuracy of modeling is the main bottleneck in implementing CAD tools for the first pass design of 
the RF/microwave circuit. Accurate and reliable characterization of RF/microwave circuit component is one of the basic prerequisites of a successful CAD. Fortunately, the emerging of electromagnetic (EM) simulation techniques helps to make significant improvement on accurate modeling. Various techniques for EM field simulation [36-38] have been developed. Several commercial EM simulators have been built using these EM simulation techniques. We can single out the High Frequency Structure Simulator (HFSS) from Ansoft and the MoM product from Sonnet software. These EM simulators can offer excellent accuracy if critical areas of the EM structures are meshed with a sufficiently small grid. However, a major disadvantage of the EM simulators is their high demands for computer resources. They are computationally intensive, which limits their application in RF/microwave circuit design. For this reason, lumped element models, closed form expressions and analytical models have been derived from EM simulation to shorten the computing time. However, these derived models are valid only within a restricted range, beyond which these fast models may not provide sufficiently accurate solutions.

As mentioned in Chapter 1, the use of ANNs has provided a powerful approach for developing RF/microwave models. Significant advances have been made in the exploration of the neural network as an alternative vehicle for EM-based design in the RF and microwave areas. ANNs allow fast and accurate model development even if component formulae or equivalent circuits are unavailable. Neural network possesses the ability of learning from samples of input-output data to accurately model nonlinear relationships. It has the distinguished advantage of being fast and accurate. In the past 
few years, ANNs have been widely used in RF/microwave circuit design. Various applications have been made by using ANNs, such as microstrip interconnects [31], vias [18], spiral inductors [1], FET devices [31, 11], HBT devices [40], HEMT devices [41], filters [42], amplifiers [39, 43], coplanar waveguide (CPW) circuit components [21], mixers [43], antennas [44], embedded resistors [44-47], packaging and interconnects [16], and etc. Neural networks have also been used in circuit simulation and optimization [39, 48], signal integrity analysis and optimization of VLSI interconnects [16, 49], microstrip circuit design [9], process design [50], synthesis [51], and microwave impedance matching [8]. These pioneering works have established the framework of the neural modeling techniques in both device and circuit levels of microwave applications.

\subsection{Neural Network Model Development Overview}

A neural network without training can not represent any device/circuit behaviour. In this section, we will review the process of developing a neural network model. At the initial stage of the neural network model development, a proper neural network structure needs to be selected first to make the model development efficient. There are various types of neural network structures: multilayer perceptrons (MLP), radial basis function (RBF) network, wavelet neural network, self-organizing maps (SOM) and recurrent neural networks. The most popular configuration is MLP which we will discuss in details later in Section 2.3.

Four major steps-problem identification, data generation, training and testing-are involved in developing a neural network model. The model development process is shown in Fig. 2-1. The first step is the identification of the model inputs and outputs 
according to the purpose of this model. The next step is the data generation which generally involves using a data generator to obtain the output for each input sample. The total amount of samples to be generated is chosen so that the developed neural model can best represent the original problem. Before the data generation, data ranges used to develop the neural network model have to be defined depending on the type of application of the target problem. Once the data ranges are determined, two sets of data, which are training data and test data, need to be generated. The training data are sampled slightly beyond the model utilization range while the test data are within the determined range. Either detailed EM simulation software or direct measurement can be used for data generation. The choice of data generators depends upon both the applications and the availability of the data generators. After the training data and test data are generated, training is performed using the training data to let the neural network learn the device/circuit behaviour. Since training is the most important step in neural model development, several training algorithms will be discussed in Section 2.4. After the neural network model is well trained, it is tested with the test data to evaluate the efficiency of the neural network model. 


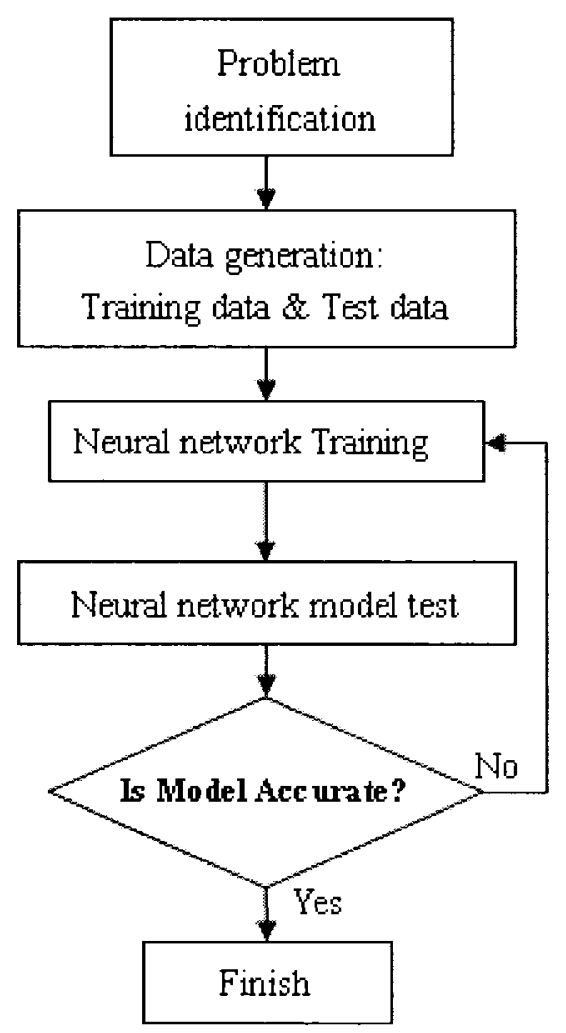

Figure 2-1. Flowchart summarizing the procedure of developing a neural network model. 


\subsection{Neural Network Structure}

Various types of neural network structures have been used for circuit design, signal processing, control, and etc. The most commonly used neural network configuration is multilayer perceptrons (MLP) [52] as shown in Fig. 2-2. The first and last layers are called input layer and output layer, respectively. The hidden layers are the layers in between.

Suppose the total number of layers is $L$. The input layer is layer 1 , the output layer is layer $L$ and the hidden layers are layer $2,3, \ldots, L-1$. Let $x_{i}$ be the $i$ th input parameter of the MLP, e.g., the geometrical/physical parameters of a microwave component. The input layer simply passes the input parameters to the hidden layer without any processing. Let the number of neurons in the $l$ th layer be $N_{l}, l=1,2, \ldots, L$. Therefore, the output of the $i$ th neuron of the lth hidden layer can be defined as $y_{i}^{\prime}$. Let $w_{i j}^{\prime}$ be the weight parameter of the link between the $j$ th neuron of the $(l-1)$ th hidden layer and the $i$ th neurons of the $l$ th hidden layer, and $\theta_{i}^{\prime}$ be the bias parameter of the $i$ th neuron of the $l$ th hidden layer. Based on these definitions, the following equations stand:

$$
\begin{gathered}
y_{i}^{\prime}=x_{i} i=1,2, \ldots, N_{1}, \\
y_{i}^{\prime}=\sigma\left(\sum_{j=1}^{N_{t-1}} w_{l j}^{\prime} \cdot y_{j}^{l-1}+\theta_{i}^{\prime}\right), i=1,2, \ldots, N_{l} \quad l=2,3, \ldots, \mathrm{L},
\end{gathered}
$$

where $\sigma(\square)$ is usually in the form of activation functions. There are three activation functions can be used for the hidden neurons [59]. The most commonly used function is 
the sigmoid function. Other possible hidden neuron activation functions are arc-tangent function and hyperbolic-tangent function.

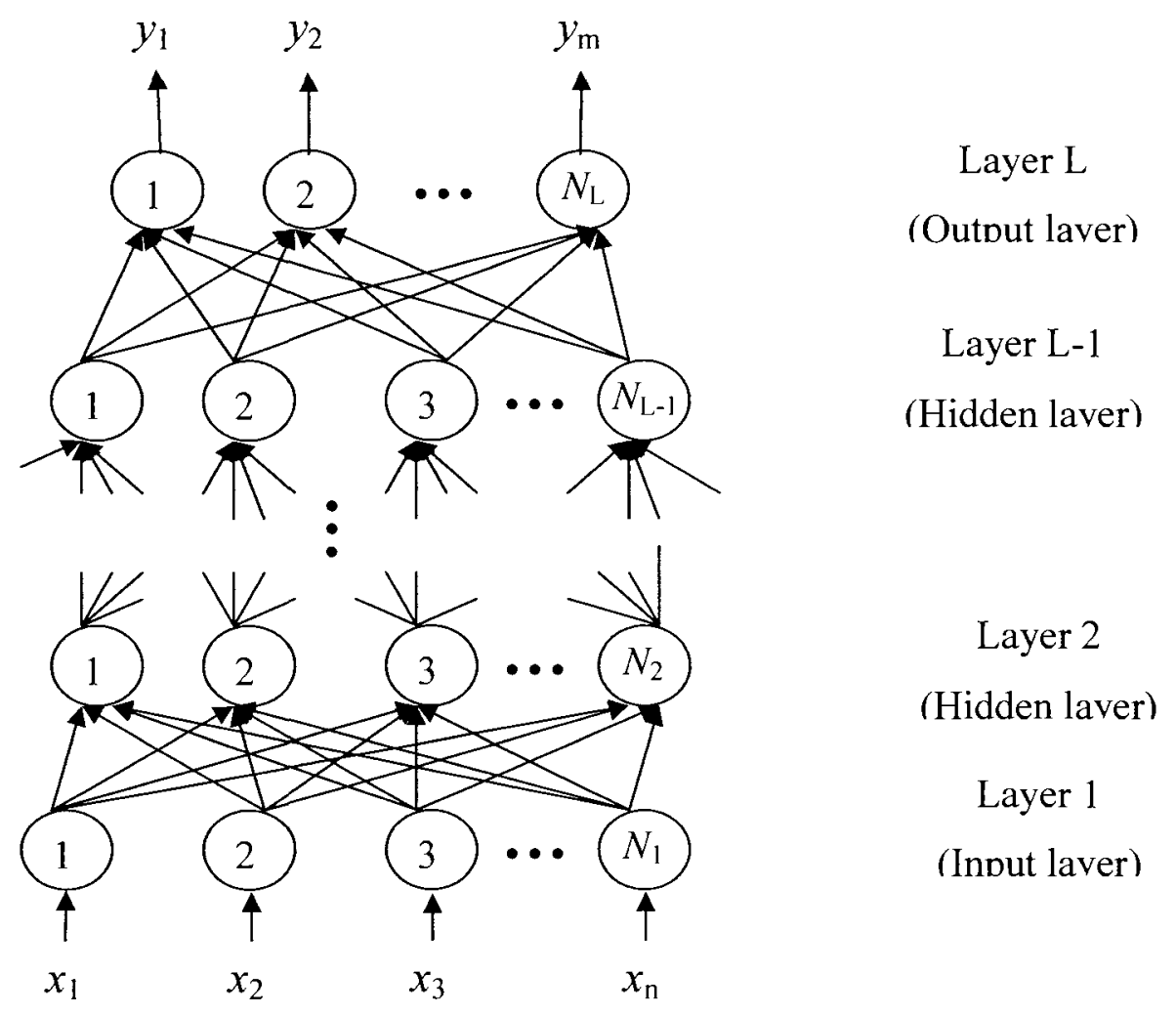

Figure 2-2. The multilayer perceptrons (MLP) structure (from Q. J. Zhang, K. C. Gupta and V. K. Devabhaktuni [52]). 


\subsection{Neural Network Training Algorithm}

The objective of training is to adjust neural network weight parameters such that the outputs of the neural network model can best match those of the training data. Only a trained neural model can represent device/circuit behaviour and be used during the design process to provide instant answer to target problem.

As described in [59], an error function is defined as the main criterion to direct weight parameters update. Consider a target problem with an input $n$-vector $\boldsymbol{x}$ and an output $m$ vector $\boldsymbol{y}$. The input-output relationship for the target problem can be defined as

$$
y=f(x) .
$$

Let $\boldsymbol{w}$ represent the weight parameters inside the neural network. Let the neural network model be defined as

$$
\boldsymbol{y}=f_{N N}(\boldsymbol{x}, \boldsymbol{w}) .
$$

The training data of the neural network model is denoted by the sample pair $\left(x_{k}, d_{k}\right)$ of $(x, y)$, where $x_{\mathrm{k}}$ and $\boldsymbol{d}_{\mathrm{k}}$ are samples of $\boldsymbol{x}$ and $\boldsymbol{y}$, respectively. Suppose the total sets of training data are $N_{k}, k=1,2, \ldots, N_{k}$. Based on these definitions, the error function of the training is given by

$$
E(\boldsymbol{w})=\sum_{k=1}^{N_{k}} E_{k}(\boldsymbol{w})
$$

where $E_{k}(w)$ is defined as

$$
E_{k}(\boldsymbol{w})=\left[\frac{1}{p} \sum_{j=1}^{m}\left|y_{j}\left(\boldsymbol{x}_{k}, \boldsymbol{w}\right)-d_{j k}\right|^{p}\right],
$$


where $d_{\mathrm{jk}}$ is the $j$ th element of $d_{\mathrm{k}}, y_{\mathrm{j}}\left(\boldsymbol{x}_{\mathrm{k}}, \boldsymbol{w}\right)$ is the $j$ th output of the neural network for input sample $x_{\mathrm{k}}$, and $p$ represents least $p$ th optimizations. During the training, $\boldsymbol{w}$ is updated such that a minimized error function can be achieved.

The validity of the trained neural model can be tested by another independent set of data called test data. Several error functions can be used to measure the quality of trained neural model during the test. As defined in [59], a relative error $\delta_{q j}$ for the $j$ th output of neural model on the $q$ th test data sample is given by

$$
\delta_{q \jmath}=\frac{y_{j}\left(\boldsymbol{x}_{q}, \boldsymbol{w}\right)-d_{j q}}{d_{\max , j}-d_{\min , j}}, j=1,2, \ldots, m, q=1,2, \ldots, N_{q},
$$

where $N_{q}$ is the total number of the test data. A pth-norm measure is then defined as

$$
M_{p}=\left[\sum_{q=1}^{N_{q}} \sum_{j=1}^{m}\left|\delta_{q j}\right|^{p}\right]^{1 / p}
$$

When $p=1$, the average test error can be directly calculated from $M_{1}$ as

$$
\text { Average Test Error }=\frac{M_{1}}{\operatorname{Size}\left(N_{q} m\right)}=|\bar{\delta}| \text {, }
$$

where $|\bar{\delta}|$ is the mean value of the absolute error $\left|\delta_{q u}\right|$ for all $q$ and $j$.

When $p=2$, the $p$ th-norm measure is the Euclidean distance between the neural model prediction and the test data. When $p=\infty$, the $p$ th-norm measure is the maximum test error and is also known as the worst-case error, that is

$$
\text { Worse-case Error }=M_{x}=\max _{q=1}^{N_{q}} \max _{j=1}^{m}\left|\delta_{q l}\right| .
$$


There are different training algorithms and each algorithm has its own scheme for updating the weights of neural networks. Three typical types of training algorithms are reviewed as follows.

\subsubsection{Back Propagation (BP) Training Algorithm}

The Back Propagation (BP) is one of the most popular training algorithms for neural network training. It was first proposed in 1986 [53]. BP belongs to the Gradient-based methods category and is based on the steepest decent principle [54]. The weights are updated along the steepest descent direction in which the objective function is decreasing most rapidly. Learning rate will be used to decide the size of the weights update. Although the objective function decreases most rapidly along the negative of the gradient, this does not necessarily produce the fastest convergence. As a result, two major disadvantages of the basic BP algorithm are the slow convergence and chances of weight oscillation. One possible way to enhance the stability of BP is to keep the learning rate small and add an additional term called momentum term to reduce the weight oscillation. The momentum term can control the influence of the previous updating directions of the weights on the current ones.

\subsubsection{Gradient-Based Optimization Techniques}

As the learning process of the neural network can be seen as an optimization procedure, higher order optimization methods using gradient information can be used for neural network training such that the rate of the convergence can be improved. 
From the optimization point of view, the objective function $E(\boldsymbol{w})$ is a parametric function depending on all the training parameters in the neural network. The goal of the training is to minimize $E(\boldsymbol{w})$ through optimization of the weight parameter $\boldsymbol{w}$. The main difference between different decent algorithms lies only in the update direction. The determination of the direction from the gradient of the object function $E$ is called the gradient-based descent method. Two commonly used training algorithms based on gradient-based optimization techniques, namely conjugate gradient method and QuasiNewton method are reviewed as follows.

\section{A. Conjugate Gradient Training Algorithm}

In the conjugate gradient algorithms, a search is performed along the conjugate directions, which produces generally faster convergence than along steepest descent directions. The step size is adjusted in each epoch. A line search is made along the conjugate gradient direction to determine the step size. Conjugate gradient methods are generally faster than BP and scale well with the neural network size. However, two important concerns need to be taken into consideration when applying the conjugate gradient methods to neural network training. First, as every objective function evaluation requires presenting all sample data during the neural network feedforward calculation, the one-dimensional optimization is computationally expensive. Another issue is that the error function for the neural network is not quadratic as defined in (2.6), i.e., it depends upon the degree to which a local quadratic approximation can be applied to the training error surface. 


\section{B. Quasi-Newton Training Algorithm}

The Quasi-Newton algorithm was derived from quadratic objective function. It generally can converge in fewer iterations. The inverse of the Hessian matrix in the Quasi-Newton algorithm is used to speed up the convergence rate. The inverse of the Hessian matrix is estimated from the previous gradient directions without computation. However, the storage of the approximation of the inverse Hessian matrix will be large. Suppose there are total $N$ weight parameters in the neural network. $N^{2}$ storage space will be needed for standard Quasi-Newton methods to store the approximation of the inverse Hessian matrix. Therefore, the Quasi-Newton method may not be sufficient for a large size of neural network training due to the large amount of required storage.

\subsubsection{Global Training Algorithm}

BP, conjugate-gradient method and Quasi-Newton method as discussed earlier are all gradient-based techniques. They all share the same defect of converging to a local minimum. Under this circumstance, another category of training algorithm called global optimization method is introduced to help the neural network training to escape from the traps of the local minimum and find a global minimum. This class of methods is known as random optimization techniques. They use random search elements during the neural network training. Two important methods in this class are the genetic algorithm [55] and the simulated annealing (SA) algorithm [56]. The genetic algorithms are adaptive techniques that can efficiently search the entire space and finally locate the solutions near the optimal results. The SA training algorithm is inspired by the idea of the physical 
annealing process in crystal growth and is capable of finding the global minimum by using a control parameter called temperature.

Because of the random search characteristic, training of the neural network using global optimization methods can be slow. Therefore, a hybrid method that incorporates the conjugate gradient method into the random optimization was proposed in [57]. The basic idea of this hybrid method is through switching between the conjugate gradient method and the random optimization to find a global minimum, and the same time, speed up the training process.

After reviewing the details of different types of training algorithms, we may have a better understanding of the neural network training process and can choose the type of training algorithms wisely according to the complexity of the target problem and the availability of existing computational resources.

\subsection{Knowledge Based Neural Networks}

With the continuing rapid development in neural network based RF/microwave circuit design, more accurate models are needed while the cost of design has to be reduced. The conventional pure neural network normally requires a large amount of training data to predicate the problem behaviour as it has to learn from scratch. The generation of a large amount of data can be very expensive. The use of knowledge-based neural network approaches can provide additional information of the original problem and therefore require less training data compared to the pure neural network. Meanwhile, the use of knowledge-based neural networks can also help to enhance the generalization and extrapolation capability of neural models. Several methods of combing existing 
knowledge with neural networks are described, including the knowledge-based neural networks (KBNN) method [31], source-difference method (DM) [18], the priorknowledge input (PKI) [32] method and space mapping (SM) [33] method.

\subsubsection{Knowledge-Based Neural Network (KBNN)}

The KBNN method was first proposed in [31]. The basic idea behind KBNN model development is incorporating the existing microwave knowledge into the overall neural network structure. Quite different from MLP, the KBNN structure has six layers which are known as the input layer $\mathbf{X}$, the knowledge layer $\mathbf{Z}$, the boundary layer $\mathbf{B}$, the region layer $\mathbf{R}$, the normalized region layer $\mathbf{R}^{\prime}$ and the output layer $\mathbf{Y}$. The input layer of the KBNN behaves the same as that of MLP which accepts the input parameters from outside and simply passes them to the next layer. The knowledge layer is the place where microwave knowledge is incorporated into the neural network in the form of empirical or semi-analytical functions. The boundary layer can incorporate the knowledge in the form of problem-dependent boundary functions, or behave like linear boundaries in the absence of boundary knowledge. The region layer contains neurons to construct regions from boundary neurons. The normalized region layer consists of neurons based on rational functions, which are used to normalize the outputs of the region layer. The output layer contains second-order neurons combining knowledge neurons with normalized region neurons. 


\subsubsection{Source Difference Method (DM)}

DM was first proposed in [14]. DM is based on the idea of combining RF/microwave empirical and equivalent circuit together with neural network learning process. It is also known as a hybrid EM-ANN model. The overall structure of DM consists of two parts: the prior knowledge in the form of an empirical or equivalent circuit model and the neural network. The neural network is used to learn the difference between accurate EM data from either detailed EM analysis or direct measurement and approximating model. This method is expected to produce accurate solutions efficiently only if the difference between accurate data and approximate model has a smooth input-output relationship. It should be noticed that if the mapping from the difference between accurate data and outputs of the approximate model is not simpler than the original problem mapping, the benefit from this DM method will be little.

\subsubsection{Prior Knowledge Input (PKI) Method}

The PKI method was first proposed in [23]. The PKI method is also based on the idea of embedding the prior knowledge in the form of the empirical model or the equivalent circuit into the neural network. In the PKI method, the outputs of the approximate model are used as the inputs of the neural network to represent the available knowledge while the target problem inputs are also added to the inputs of the neural network. In this case, the mapping that the neural network needs to learn is from both the outputs of the approximate model and the inputs of the target problem to the desired output data. By

including the original problem inputs as additional model inputs to the neural network, 
the mapping is considerably simplified. Therefore, less training data are required compared to the need of training data for a pure neural network without prior knowledge.

\subsubsection{Space Mapping (SM) Method}

The SM neural network model is based on the space mapping concept which was first proposed by Bandler et al. [33]. Space mapping is a concept for circuit design that combines the computational efficiency of "coarse" models with the accuracy of "fine" models. The coarse models are typically empirical functions or equivalent circuit models, which are computationally efficient. However, the coarse model is valid only within a limited range, beyond which the model prediction may not be trustworthy. On the other hand, fine model, e.g., CPU intensive EM simulation, can provide accurate predication, but can be very expensive. The SM concept, which is inspired by the trade-off between the speed and the accuracy of current existing techniques, establishes a mathematical link between the coarse model and the fine model. It directs the bulk of CPU intensive evaluations to the coarse model, while preserving the accuracy of the fine model. The SM technique is frequency dependent. The operating frequency can also be included in the mapping function, which allows us to simulate the coarse model at different frequencies. 


\subsection{Conclusions}

In this chapter, the need of the ANNs in $\mathrm{RF} /$ microwave circuit design has been reviewed first. As pointed out at the beginning of this chapter, in the conventional CAD process, detailed EM simulation is accurate but computationally intensive while the empirical model is fast but lacks of accuracy. The ANNs based approach, which can represent the target problem accurately once trained, is very much needed to provide a powerful alternative to the design of the modern RF/microwave circuit. However, most pure neural network needs a large amount of training data to get accurate solutions, which results in the high cost of the model development. Therefore, knowledge-based neural network has been developed to reduce the training data, and meanwhile, enhance the generalization and extrapolation capability of the neural network models. 


\section{Chapter 3:}

\section{A Combined Approach of the Transfer Function and the Neural Network (TF-NN) for Passive Microwave Components Modeling in Multilayer Circuits}

\subsection{Introduction}

With the increasing complexity and higher operating frequencies, the conventional electrical models for modern microwave design are not accurate anymore. With electromagnetic (EM) effects playing an important role in microwave/RF circuit design, models with continuous physical/geometrical information also need to include EM effects [58] during design process. As a result, accurate and efficient characterizations of microwave components modeling need to be carried out. The conventional ComputerAided Design (CAD) approaches such as full-wave analysis can lead to the accurate results, but at much higher computational expense which prevents their use in practical interactive $\mathrm{CAD}$ [14]. Furthermore, for high-level circuit design, the microwave component models should vary continuously both with frequencies and geometrical 
and/or electrical parameters. Therefore, modeling technique that can provide fast parametric EM models with continuously varying geometrical parameters are essential.

The most popular approach for passive microwave component modeling is using equivalent circuits to capture the EM behaviour of the target problems. However, the equivalent circuits may become inaccurate if the geometrical and/or physical parameters of the components and frequency of interest change. Furthermore, the equivalent circuit may not be applicable if a new device is introduced. Therefore, equivalent circuits are not sufficient in terms of accuracy and may involve manual effort in determining model structures.

As described in Chapter 2, artificial neural networks (ANNs) modeling approach has been studied for microwave modeling and design $[59,60]$ as a powerful alternative to the conventional approach. Once trained with reliable learning data, the neural network can then represent the model within the range of trained physical and/or electrical parameters, avoiding otherwise manual effort of developing equivalent circuit topology.

However, since the target problem can be highly nonlinear and multi-dimensional, such neural network model may require more training data and more hidden neurons to learn the EM behaviour, which may become expensive if a large amount of the training data are needed. This chapter presents a methodology that can accurately model complex passive microwave components with limited training data. In the TF-NN method, neural network is first used to map the geometrical parameters of the passive microwave components into the coefficients of the transfer function. The transfer function then produces the overall model outputs. As the transfer function used in this method contains 
prior knowledge of the target problem, the neural network mapping is significantly improved with the embedded prior knowledge, and therefore, less training data are needed. EM simulation data are used to develop this TF-NN model. Inputs of the combined model are geometrical/physical parameters of the passive microwave components while outputs of the model are the corresponding EM behaviour. The TF-NN model preserves the accuracy of detailed EM simulation and can be much faster than the EM simulators. Comparisons of CPU time using TF-NN model and EM simulators will be shown in the application examples, which are via model for integrated circuits, via model for printed circuit boards and multilayer square inductor model. Through these examples, this accurate and fast TF-NN model can be proved to be efficient and feasible in passive microwave component modeling.

\subsection{Combined Approach of Transfer Function and Neural Network (TF-NN)}

\subsubsection{Neural Network (NN) Model: Problem Statement}

Let $\boldsymbol{x}$ represent a $N_{\mathrm{x}}$-vector as the neural network inputs, which contains geometrical parameters of passive microwave components. Let $\boldsymbol{y}$ represent a $N_{\mathrm{y}}$-vector as neural network outputs, which contains the response of the components under consideration, e.g., S-parameters. The physics/EM relationship between $\boldsymbol{x}$ and $\boldsymbol{y}$ can be highly nonlinear and multi-dimensional. It can be defined as

$$
y=f(x) .
$$


Let $\boldsymbol{w}$ be a vector containing all the weight parameters representing the interconnections between the model inputs and outputs. The definition of $\boldsymbol{w}$ and the manner in which $y$ is computed from $\boldsymbol{x}$ and $\boldsymbol{w}$ determine the structure of neural network. Let the neural network model be defined as

$$
y=f_{N N}(x, w)
$$

The most popular neural network structure is the feedforward multilayer perceptrons (MLP) where neurons are grouped into layers. For a given input, some neurons are turned on, some are off, and some are in transition. It is this combination of neuron switching states that enables the neural network to learn the input-output relationship. The microwave component modeling problems can be described as a nonlinear relationship between input vector $x_{\mathrm{j}}$ and output vector $\boldsymbol{d}_{\mathrm{j}} . \boldsymbol{x}_{\mathrm{j}}$ and $\boldsymbol{d}_{\mathrm{j}}$ can be grouped together as the training data: $\left(\boldsymbol{x}_{\mathrm{j}}, \boldsymbol{d}_{\mathrm{j}}\right)$, where $\boldsymbol{x}_{\mathrm{j}}$ is a sample of $\boldsymbol{x}, \boldsymbol{d}_{\mathrm{j}}$ represents output $\boldsymbol{y}$ for given input $\boldsymbol{x}_{\mathrm{j}}$. Let $T$ be the index set of training data and $j$ be the sample index. By setting $p=1$ in (2.6), The error function during training can be expressed as

$$
E(\boldsymbol{w})=\frac{1}{2} \sum_{j \in T} \sum_{k=1}^{N_{y}}\left(y_{k}\left(\boldsymbol{x}_{j}, \boldsymbol{w}\right)-d_{j k}\right)^{2},
$$

where $d_{\mathrm{jk}}$ is the $k$ th element of $\boldsymbol{d}_{\mathrm{j}}, y_{\mathrm{k}}\left(\boldsymbol{x}_{\mathrm{j}}, \boldsymbol{w}\right)$ is the $k$ th output of the neural network for input sample $\boldsymbol{x}_{\mathrm{j}}$. The purpose of training is to adjust $\boldsymbol{w}$ such that the error function $E(\boldsymbol{w})$ is minimized. At the end of training, this neural network can represent a general linear/nonlinear relationship and be used online during passive microwave components design providing fast model evaluation in replace of the original slow model from the EM simulators. However, when the modeling problems become highly nonlinear and multi- 
dimensional, learning of the neural network becomes difficult, which means it may need more data points to cover the entire information of the target problems. Therefore, it is necessary to exploit an efficient way to learn the complex input-output relationships.

\subsubsection{Combined Approach of Transfer Function and Neural Network (TF-NN) Development}

The combined TF-NN model is aimed at improving this situation. This model uses coefficients of the transfer function as an intermediate step to enhance the learning ability of the neural network. Structure of this combined model is shown in Fig. 3-1. It is a two level structure: The lower level is neural network model while the higher level is the transfer function.

MLP is selected as the neural network structure to map the geometrical/physical parameters into the coefficients of the transfer function. As the components we used in application examples have the characteristic that $S_{21}$ equals to $S_{12}$, the following three transfer function equations are adequate to represent $S_{11}, S_{21}, S_{22}$, respectively,

$$
\begin{aligned}
& H_{1}(s)=\frac{b_{0}+b_{1} s+\ldots+b_{n-1} s^{n-1}}{a_{0}+a_{1} s+\ldots+a_{n-1} s^{n-1}+a_{n} s^{n}}, \\
& H_{2}(s)=\frac{c_{0}+c_{1} s+\ldots+c_{n-1} s^{n-1}}{a_{0}+a_{1} s+\ldots+a_{n-1} s^{n-1}+a_{n} s^{n}}, \\
& H_{3}(s)=\frac{d_{0}+d_{1} s+\ldots+d_{n-1} s^{n-1}}{a_{0}+a_{1} s+\ldots+a_{n-1} s^{n-1}+a_{n} s^{n}},
\end{aligned}
$$


where $a_{0}=1, s=j^{*} \omega, \omega$ is the angular frequency, $n$ is the number of effective order of the transfer functions. Inputs of the MLP are the geometrical/physical parameters of the microwave components and outputs of the MLP are the coefficient values:

$$
\left\{a_{i}, i \in(1,2,3 \cdots n)\right\},\left\{b_{i}, i \in(0,2,3 \cdots n-1)\right\},\left\{c_{i}, i \in(0,2,3 \cdots n-1)\right\} .\left\{d_{i}, i \in(0,2,3 \cdots n-1)\right\} .
$$

\section{EM behaviour}

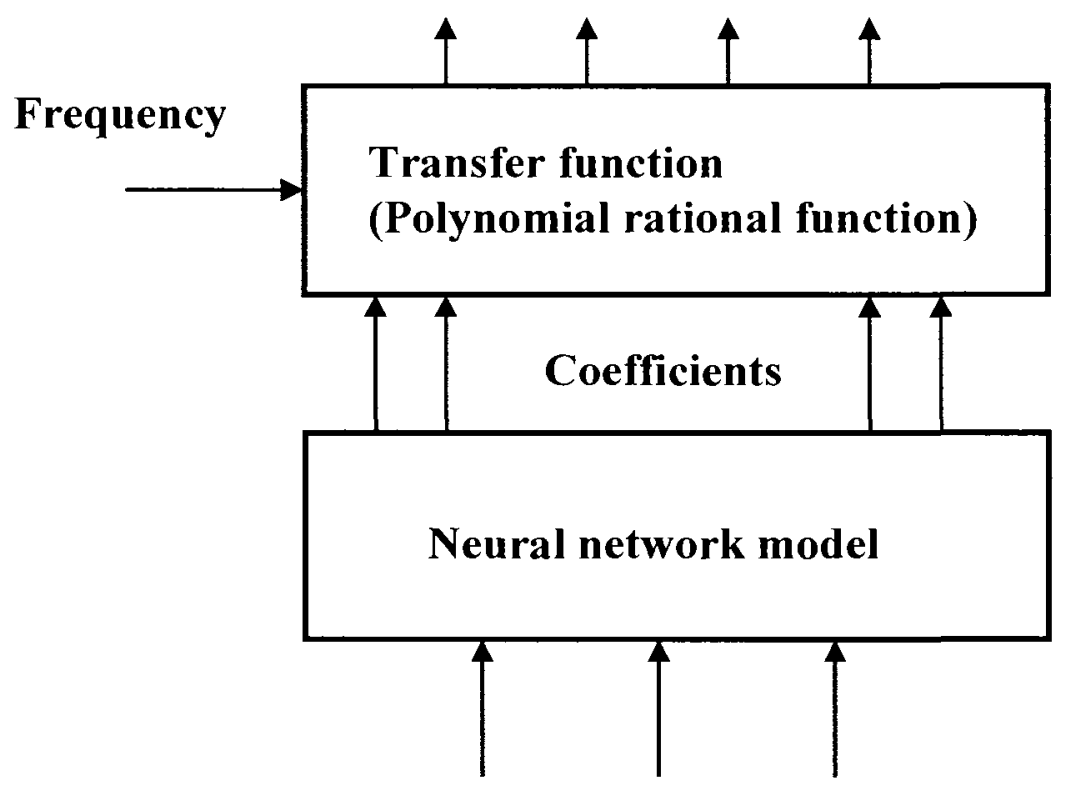

Geometrical/Physical parameters

Figure 3-1. Structure of the TF-NN model: It is a hierarchical model containing two levels: The higher level is transfer function equations while the lower level is neural network model. 
Neural network model is trained to learn this nonlinear input-output relationship. EM data with different geometrical structures can be generated from the full wave EM simulator. The EM data contain the S-parameters with respect to the different geometrical structures. The coefficients of the transfer function can be achieved by using the vectorfitting method $[61,62]$ : For frequency bandwidth of interest, the method can compute the coefficients of the transfer function with real and imaginary parts of S-parameter information.

We can define the geometrical parameters of a target passive microwave component as a $N_{\mathrm{p}}$-vector $\boldsymbol{x}$. The coefficients can be defined as

$$
\boldsymbol{g}_{c}=\left\{a_{1}, a_{2} \cdots a_{n}, b_{0}, b_{1} \cdots b_{n-1}, c_{0}, c_{1} \cdots c_{n-1}, d_{0}, d_{1} \cdots d_{n-1}\right\}
$$

By setting $p=2$ in (2.6), error function for the MLP training can be redefined as

$$
E(\boldsymbol{w})=\frac{1}{2} \sum_{i \in T} \sum_{k=1}^{N_{p}}\left(f_{N N_{k}}\left(\boldsymbol{x}_{i}, \boldsymbol{w}\right)-g_{c k}\right)^{2},
$$

where $\boldsymbol{w}$ is a vector of weights, $\boldsymbol{x}_{\mathrm{i}}$ is a sample of vector $\boldsymbol{x}, g_{\mathrm{ck}}$ is the $k$ th element of $\boldsymbol{g}_{\mathrm{c} \cdot} f_{N N_{k}}$ is the $k$ th output of the neural network for the given input sample $\boldsymbol{x}_{\mathrm{i}}$. This neural network structure is shown in Fig. 3-2. 


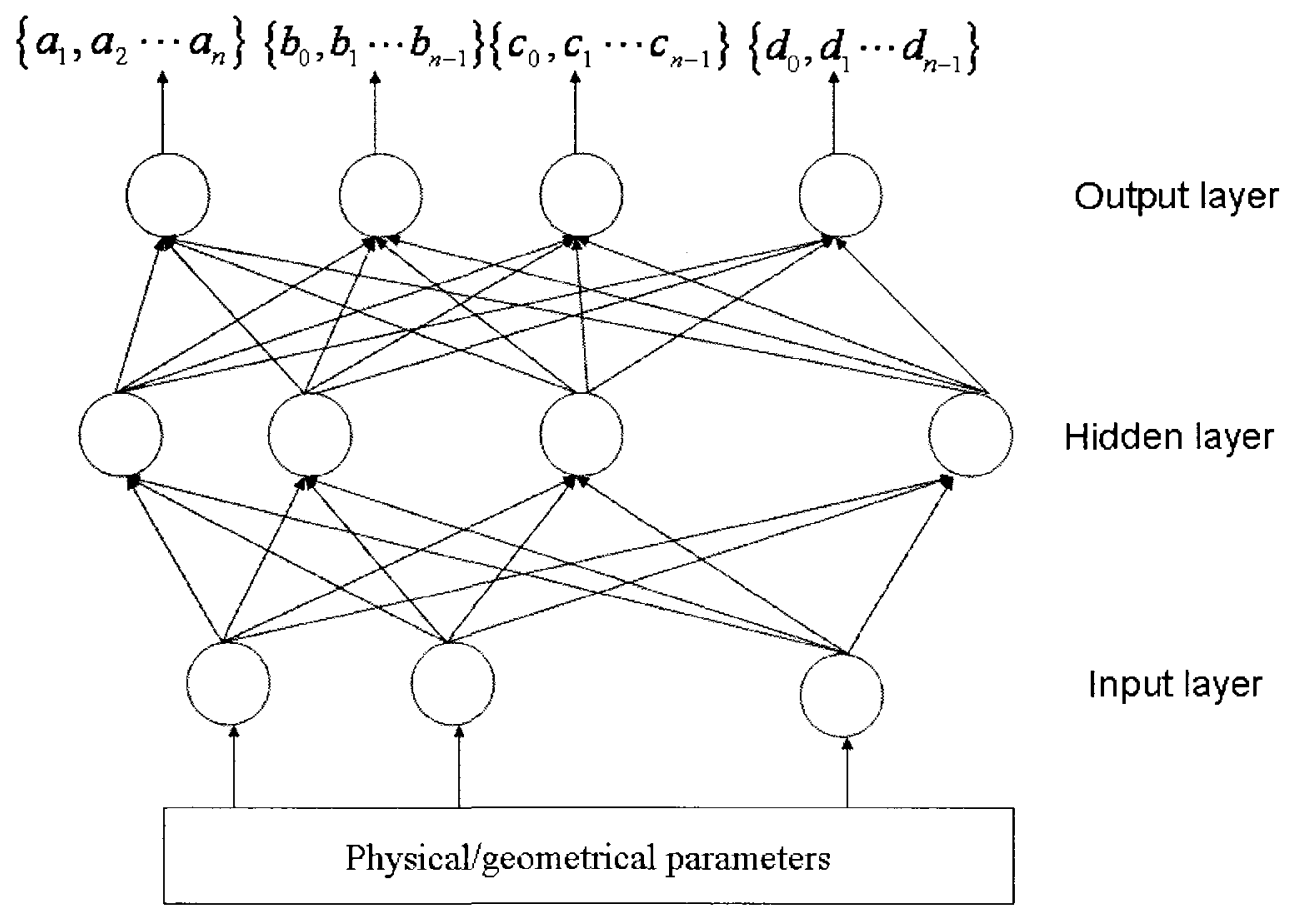

Figure 3-2. MLP neural network structure used in the TF-NN model.

Training of the neural network model can be done through NeuroModeler [63] software. After being trained, the neural network model is ready to be combined with the transfer function. The coefficients learned by the neural network are then supplied to the transfer function. Using these coefficient values, transfer function can compute the corresponding EM simulation results (e.g., S-parameters) as the outputs of the overall TF-NN model. In the test phase, an independent set of data containing new geometrical parameter values (i.e., never used in training) versus EM behaviour is generated using the EM simulator. This set of data is used to test the accuracy of the combined TF-NN model. 


\subsection{Application Examples for Passive Microwave Components Modeling Using the TF-NN Model}

In this section, three examples for multilayer circuit component modeling are presented to demonstrate the validity and efficiency of the TF-NN modeling approach.

\subsubsection{Via Model for Integrated Circuits}

In this example, the 3D structure of the multilayer via for integrated circuits is shown in Fig. 3-3. The EM data of this via are automatically generated from the EM simulator ADS Momentum [64]. As depicted in Fig. 3-3, the model inputs are the diameter $(D)$ of the via, the height $(H)$ of the substrate and the frequency $(F)$. As this via structure is symmetric, $S_{12}$ equals $S_{21}$ and $S_{11}$ equals $S_{22}$. Data ranges of all the parameters used in developing the TF-NN model are shown in Table 3-I. The Frequency data are only used for computing coefficients of the transfer function and are not used in training.

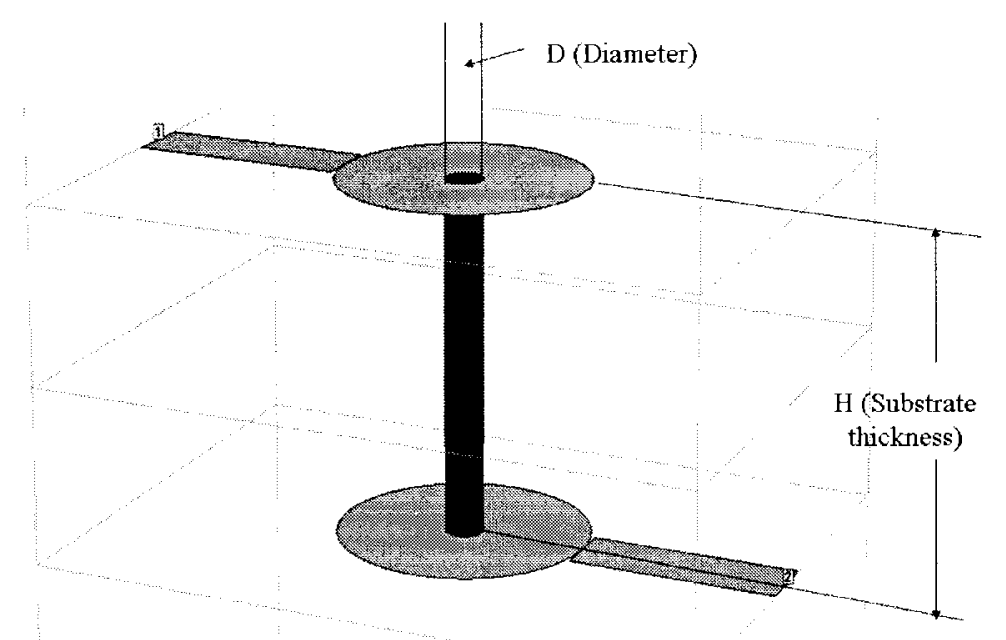

Figure 3-3. 3D structure of the via for integrated circuits. $D$ and $H$ are the design variables. 
Table 3-I. Data ranges of all the parameters used in developing the TF-NN model for the via in the integrated circuits.

\begin{tabular}{|c|c|c|}
\hline \multirow{2}{*}{ Parameters } & \multicolumn{2}{|c|}{ Data ranges of all the parameters } \\
\cline { 2 - 3 } & Min & Max \\
\hline D $(\boldsymbol{\mu m})$ & 60 & 130 \\
\hline $\mathbf{H}(\boldsymbol{\mu m})$ & 230 & 290 \\
\hline F $(\mathbf{G H z})$ & 0.25 & 20 \\
\hline
\end{tabular}

The number of the hidden neurons used in the neural network is 13. After being trained, the neural network is tested with another set of data that are within the range of training data, but never used in training. The comparisons between the outputs of the TFNN model and the EM data are shown in Fig. 3-4: Curve a and curve b show the comparisons of real part of $\mathrm{S}_{11}$ and $\mathrm{S}_{21}$ based on $D=0.29 \mathrm{~mm}, H=0.075 \mathrm{~mm}$ and $D=$ $0.23 \mathrm{~mm}, H=0.105 \mathrm{~mm}$, respectively. Table 3 -II shows the training errors and test errors of the combined model. The order of the transfer function for this via model is 8 . 

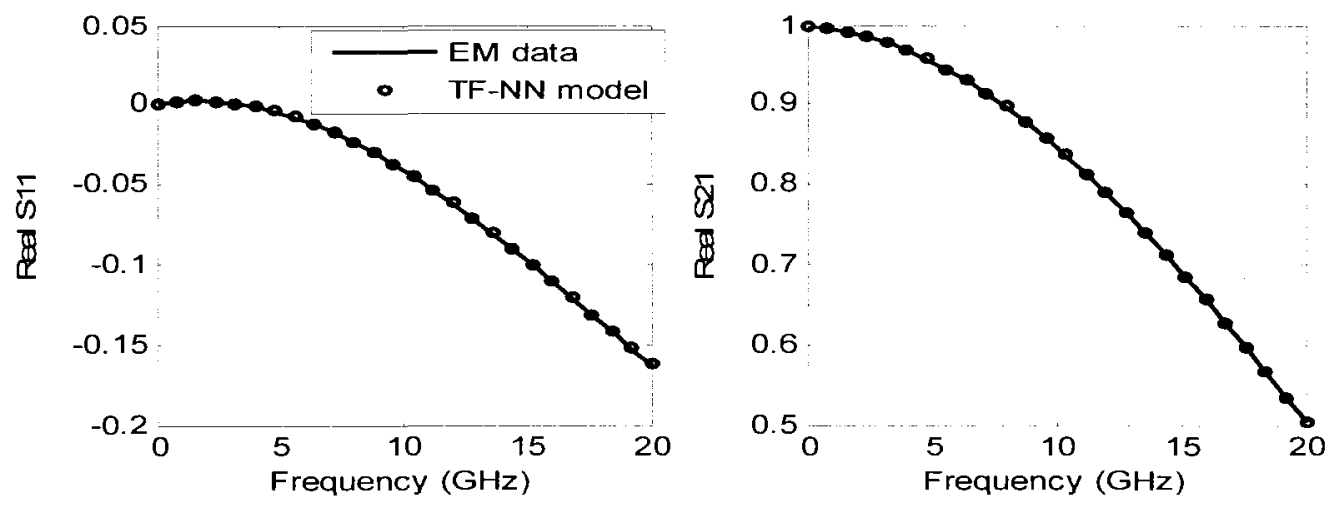

(a)
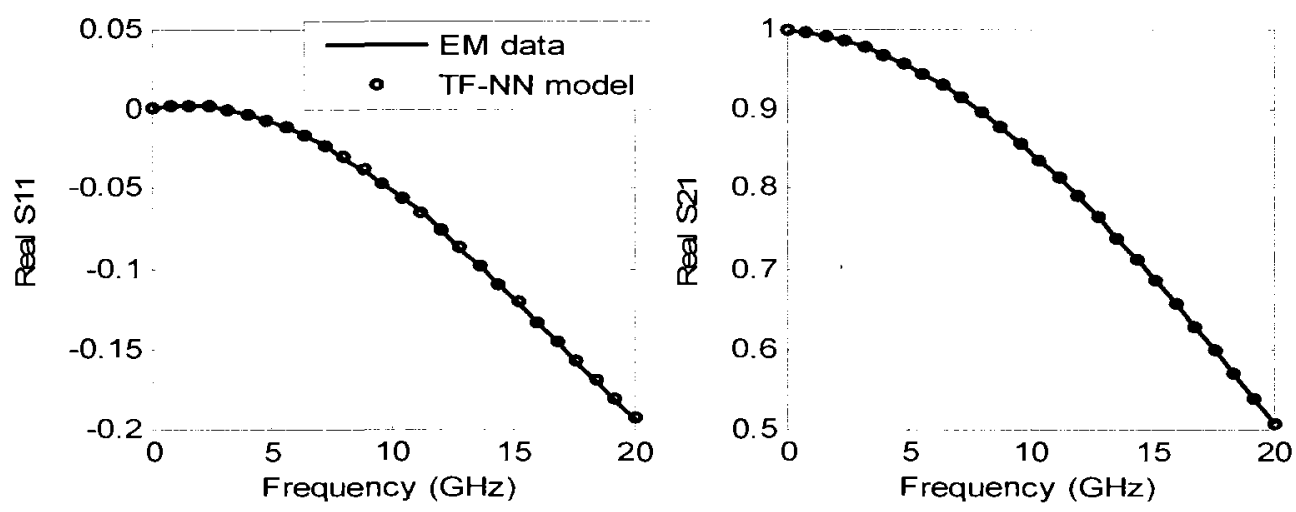

(b)

Figure 3-4. Test results of the via model for integrated circuits: (a): Test results are generated based on $H=0.29 \mathrm{~mm}, D=0.075 \mathrm{~mm}$. (b): Test results are generated based on $H$ $=0.23 \mathrm{~mm}, D=0.105 \mathrm{~mm}$. 
Table 3-II. Training/test errors for the via model in the integrated circuits

\begin{tabular}{|l|l|l|}
\hline & Training error & Test error \\
\hline Average error & $0.06 \%$ & $0.6 \%$ \\
\hline
\end{tabular}

\subsubsection{Via model for Printed Circuit Boards}

Another via example is developed to further demonstrate the efficiency of the combined TF-NN modeling approach. A three-layer via with a ground plane in the middle is shown in Fig. 3-5.

As shown in Fig. 3-5, the input parameters include the diameter $(D)$ of the via, the height $(H)$ of the substrate and the frequency $(F)$. Real and imaginary parts of the Sparameters are the overall model outputs, which are generated from the EM simulator Sonnet [65]. 19 hidden neurons are used for the neural network training. The order of the transfer function is 8 . The training of these two via examples is done through NeuroModeler. The data ranges of all the parameters used in developing the TF-NN model are shown in Table 3-III. The frequency data are only used for computing the coefficients of the transfer function and are not used in training. A good agreement is achieved between the outputs of the TF-NN model and the corresponding EM data. 


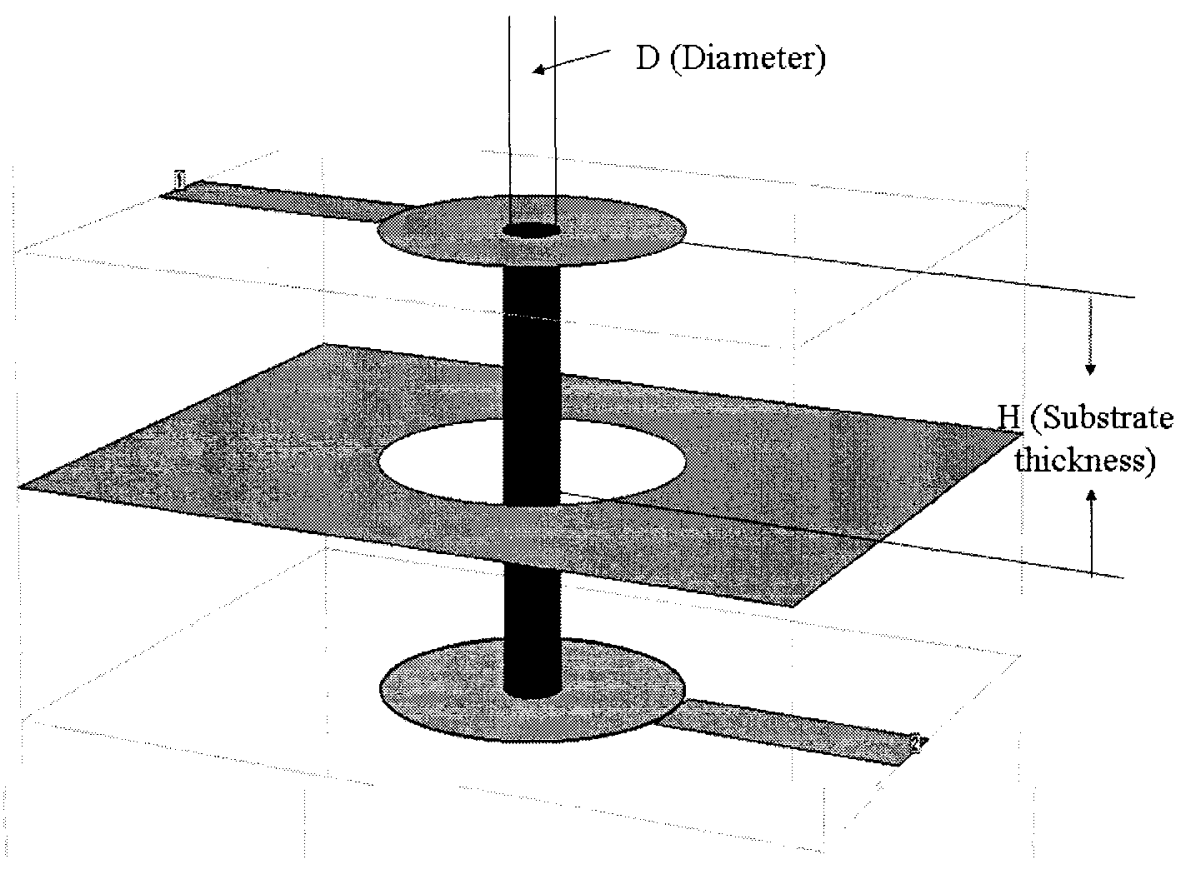

Figure 3-5. 3D structure of the via for printed circuit boards. $D$ and $H$ are the design variables.

Table 3-III. Data ranges of all the parameters used in developing the TF-NN model for the via in the multilayer circuit boards.

\begin{tabular}{|c|c|c|}
\hline \multirow{2}{*}{ Parameters } & \multicolumn{2}{|c|}{ Data ranges for all the parameters } \\
\cline { 2 - 3 } & Min & Max \\
\hline D (mm) & 0.4 & 1.4 \\
\hline H(mm) & 1.4 & 1.7 \\
\hline F (GHz) & 0.25 & 20 \\
\hline
\end{tabular}


The training errors and test errors are shown in Table 3-IV. Test results are shown in Fig. 3-6. As this via structure is symmetric, i.e., $S_{12}$ equals $S_{21}$ and $S_{11}$ also equals $S_{22}$. Curve a and curve $\mathrm{b}$ are the comparisons of the real part of $\mathrm{S}_{11}$ and $\mathrm{S}_{21}$ based on $D=0.55$ $\mathrm{mm}, H=1.5 \mathrm{~mm}$ and $D=0.77 \mathrm{~mm}, H=1.7 \mathrm{~mm}$, respectively.

Table 3-IV. Training/test errors for the via model in the printed circuit boards.

\begin{tabular}{|l|l|l|}
\hline & Training error & Test error \\
\hline Average error & $0.2505 \%$ & $0.8162 \%$ \\
\hline
\end{tabular}



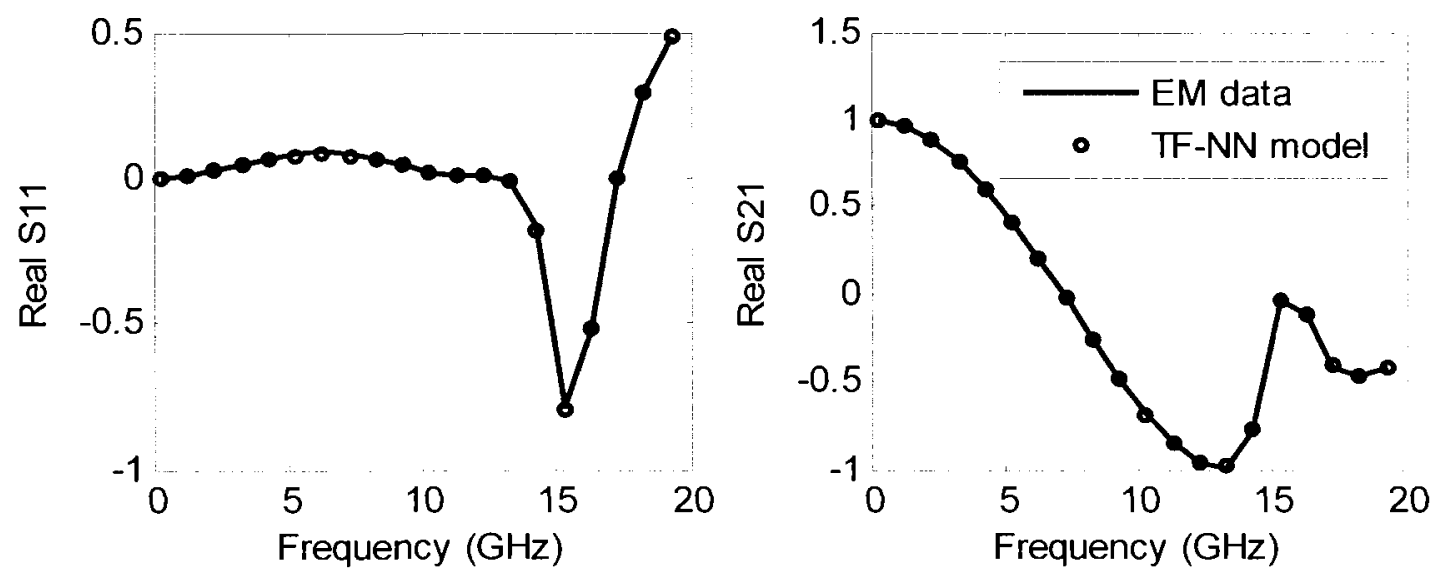

(a)
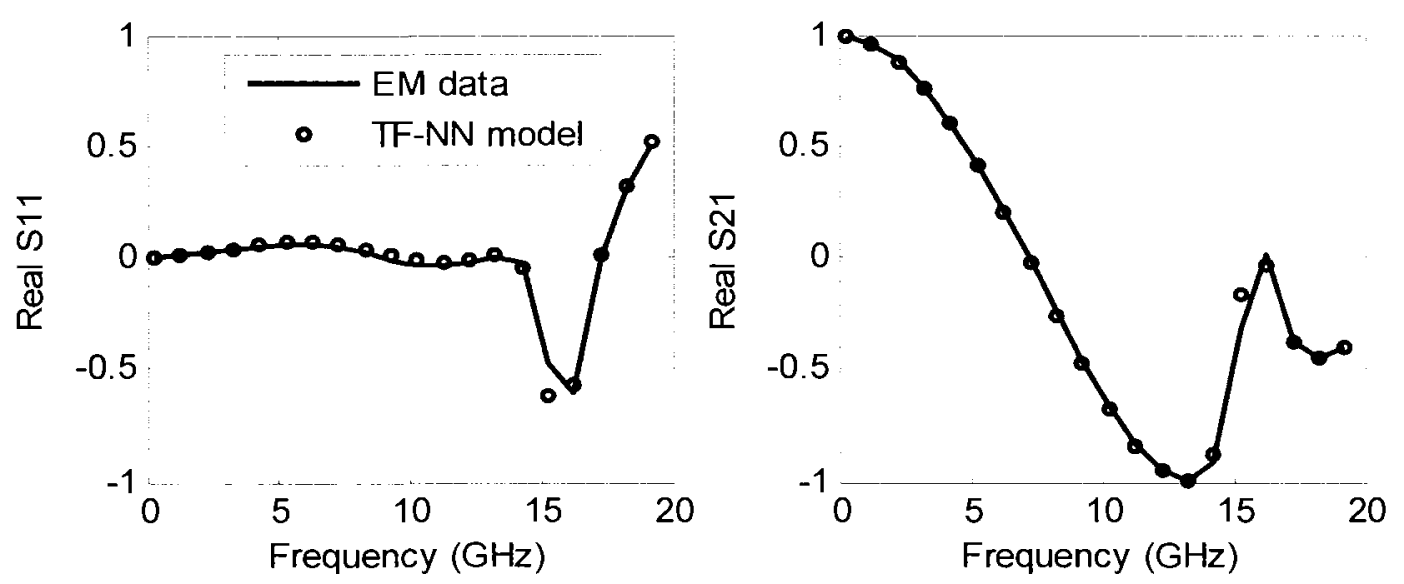

(b)

Figure 3-6. Test results of the via model for printed circuit boards: (a): The test results are generated based on $D=0.55 \mathrm{~mm}, H=1.5 \mathrm{~mm}$. (b): The test results are generated based on $D=0.77 \mathrm{~mm}, H=1.7 \mathrm{~mm}$. 


\subsubsection{Square Inductor Model}

In this example, a square spiral inductor model is developed using the TF-NN model. Fig. 3-7 shows the top view of a square inductor. This square inductor is modeled on the silicon substrate. For a typical process with two layers of metal, the top layer is used for forming the square inductor and the bottom layer is used for routing the inner turn outward. P1 is the input line while P2 is the output line. The dielectric constant for these two layers is 3.9 . The heights of the top layer and the bottom layer are $1.32 \mu \mathrm{m}$ and $2.65 \mu \mathrm{m}$, respectively.

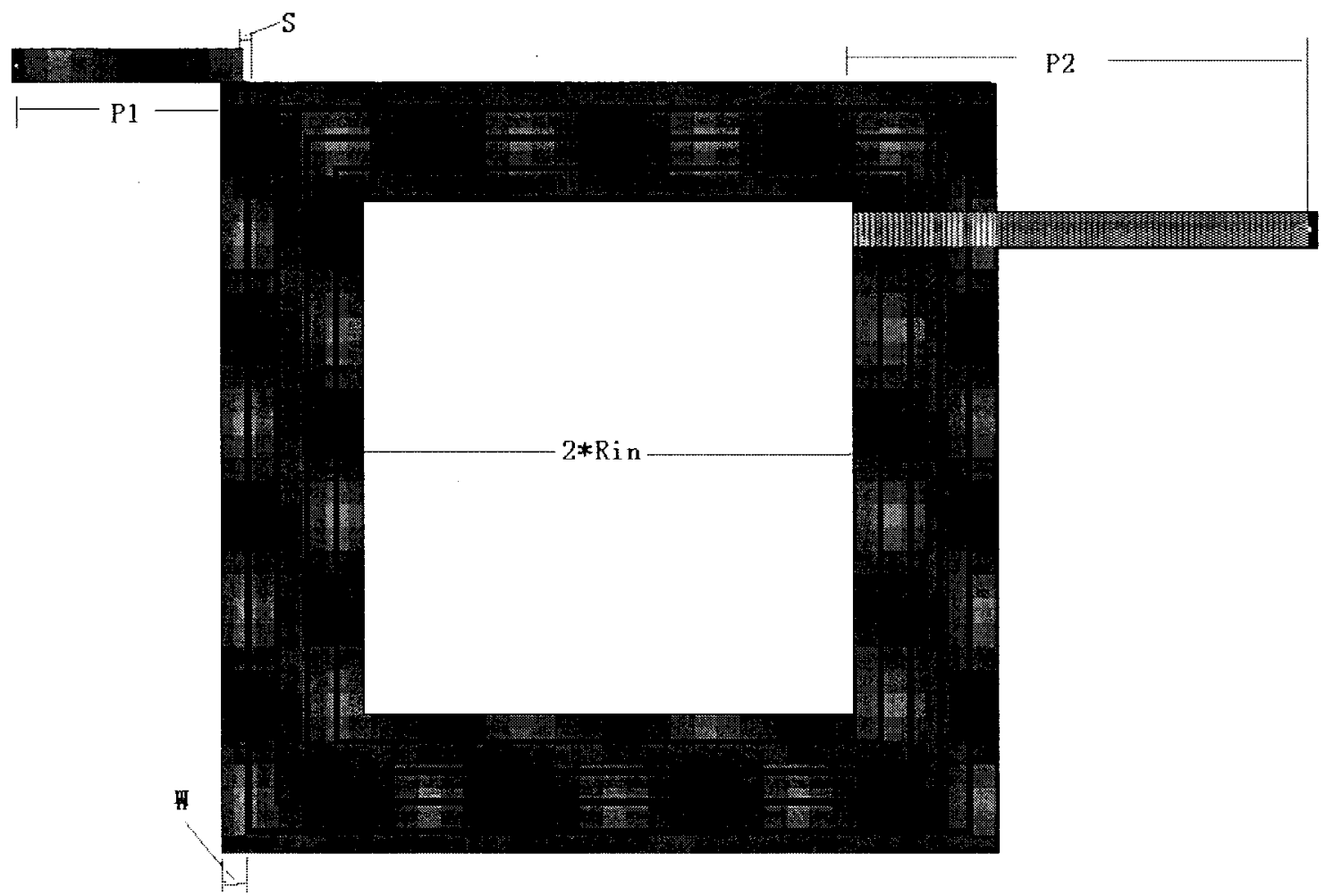

Figure 3-7. Top view of the square inductor structure with adjustable parameters. 
There are 5 inputs for this TF-NN model including the inner radius $\left(R_{i n}\right)$ of the inductor, the conductor width $(W)$, the spacing between turns $(S)$, the number of turns $(N)$ and the frequency $(F)$. As $\mathrm{S}_{12}$ equals $\mathrm{S}_{21}$, the outputs of the neural network are real and imaginary parts of S11, S12 and S22. Data ranges of all the parameters used in developing the TF-NN model for the square inductor are shown in Table 3-V. The frequency data are only used for computing coefficients of the transfer function and are not used in training. The EM data are generated from the EM simulator ADS Momentum [64].

Table 3-V. Data ranges of all the parameters used in developing TF-NN model for the square inductor.

\begin{tabular}{|c|c|c|}
\hline \multirow{2}{*}{ Parameters } & \multicolumn{2}{|c|}{ Training data ranges } \\
\cline { 2 - 3 } & Min & Max \\
\hline $\mathbf{N}$ & 1.5 & 2.5 \\
\hline Rin $(\boldsymbol{\mu m})$ & 30 & 175 \\
\hline $\mathbf{W}(\boldsymbol{\mu m})$ & 5 & 13 \\
\hline $\mathbf{S}(\boldsymbol{\mu m})$ & 1 & 20 \\
\hline $\mathbf{F}(\mathbf{G H z})$ & 0.1 & 10 \\
\hline
\end{tabular}


The number of hidden neurons used in the neural network training is 16 . The order of the transfer function used for this example is 6 . Testing is performed by comparing the outputs of the overall TF-NN model and the EM data, as shown in Fig. 3-8, Fig. 3-9 and Fig. 3-10. The test data are within the range of training data but never used in training. Table 3-VI shows the training errors and test errors of the TF-NN model.

Table 3-VI. Training/test errors of the square inductor model.

\begin{tabular}{|l|l|l|}
\hline & Training error & Test error \\
\hline Average error & $0.5314 \%$ & $0.6541 \%$ \\
\hline
\end{tabular}




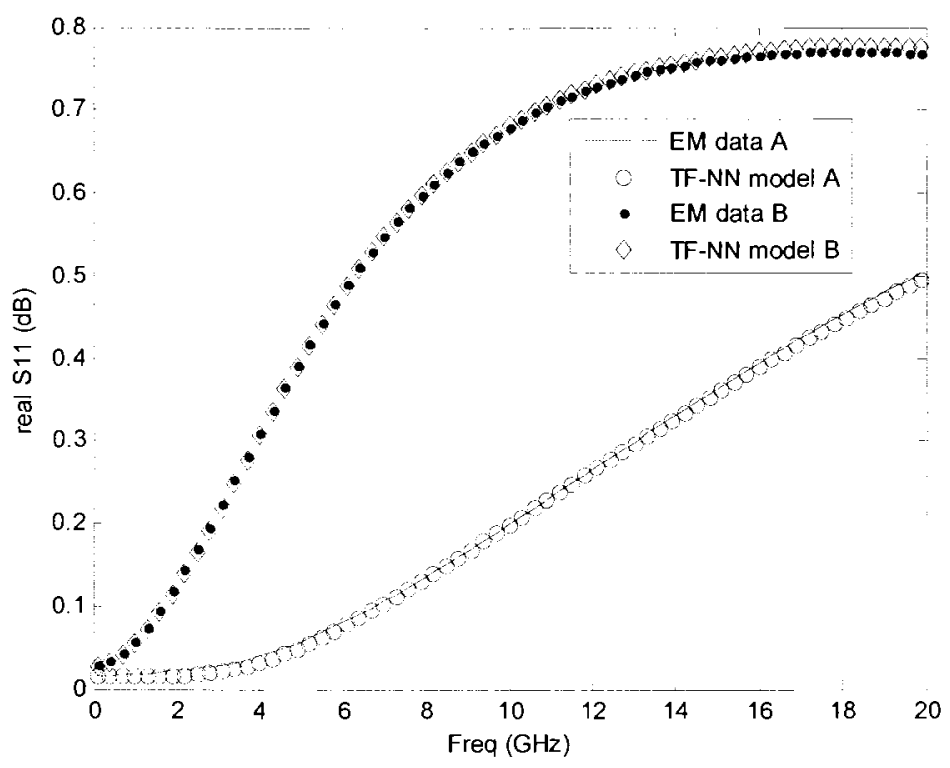

(a)

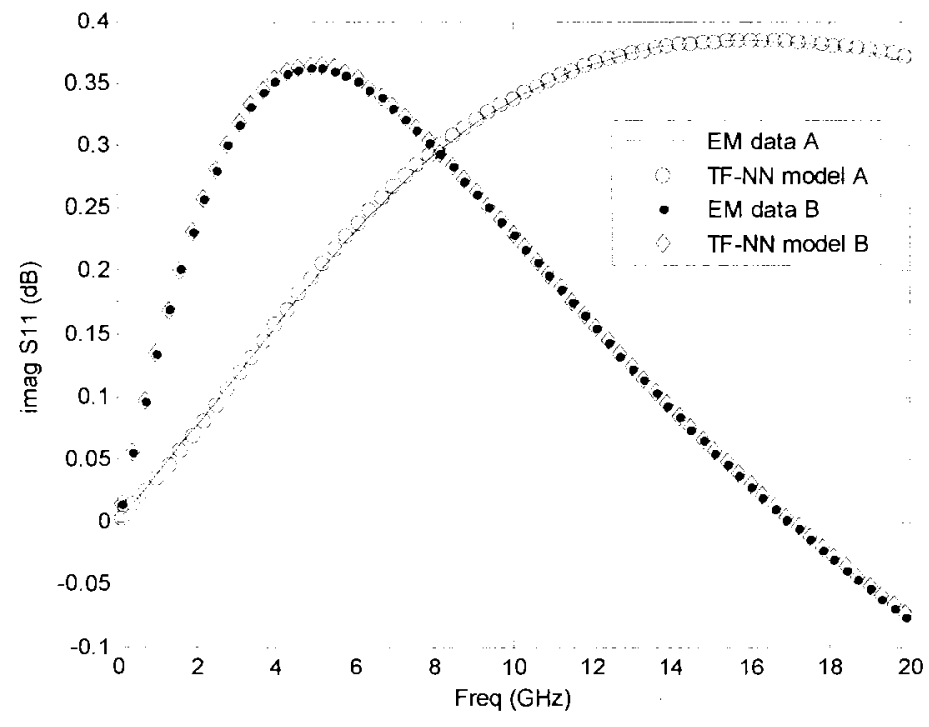

(b)

Figure 3-8. Comparisons of the real and imaginary part of the S11 for the square-spiral inductor using the TF-NN model and detailed EM simulation, Curve $A$ and Curve $B$ are generated based on $\mathrm{N}=1.5, R_{i n}=33 \mu \mathrm{m}, \mathrm{W}=6 \mu \mathrm{m}, \mathrm{S}=1.5 \mu \mathrm{m}$ and $\mathrm{N}=1.5, R_{i n}=123 \mu \mathrm{m}, \mathrm{W}=6 \mu \mathrm{m}$, $S=9.5 \mu \mathrm{m}$, respectively. The parameter values of the test structures $A$ and $B$ are never used in training. 


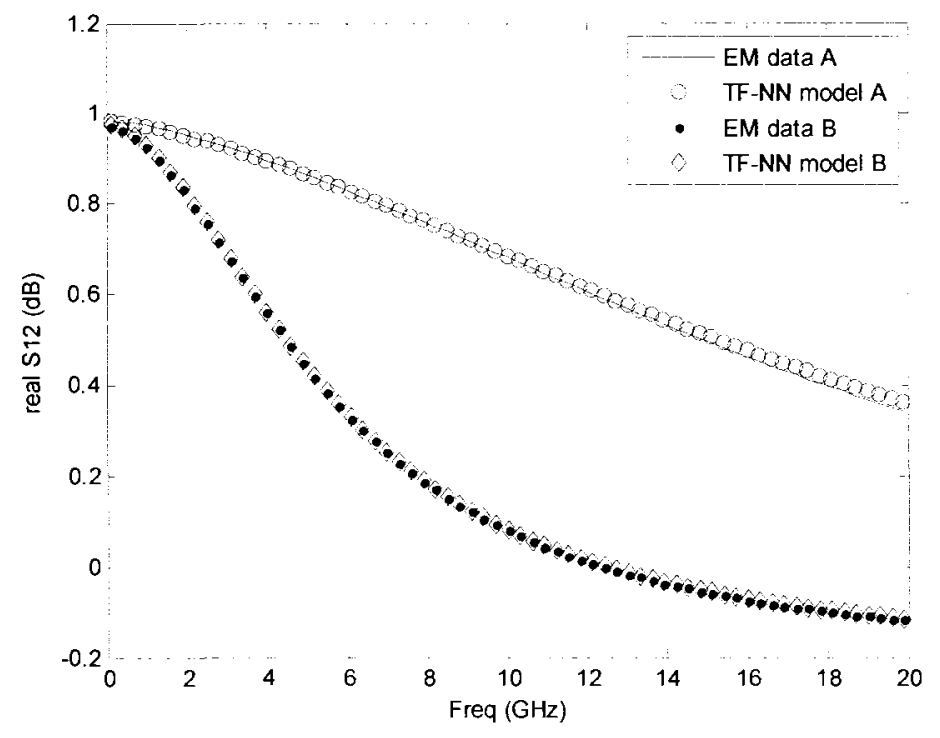

(a)

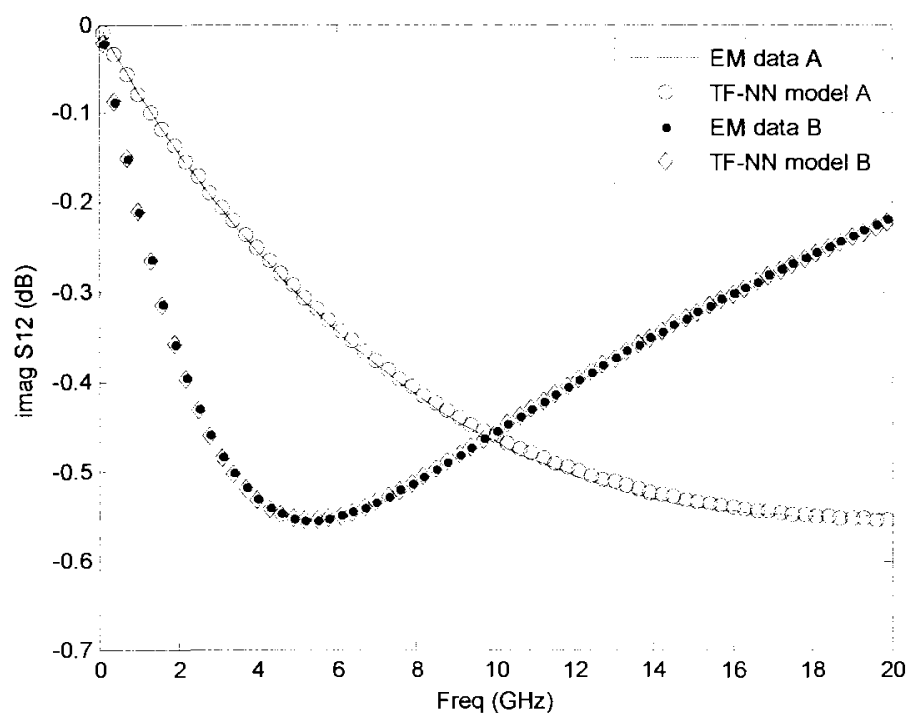

(b)

Figure 3-9. Comparisons of the real and imaginary parts of the $S 12$ for the square-spiral inductor using the TF-NN model and detailed EM simulation, Curve $A$ and Curve $B$ are generated based on $\mathrm{N}=1.5, R_{i n}=33 \mu \mathrm{m}, \mathrm{W}=6 \mu \mathrm{m}, \mathrm{S}=1.5 \mu \mathrm{m}$ and $\mathrm{N}=1.5, R_{i n}=123 \mu \mathrm{m}, \mathrm{W}=6 \mu \mathrm{m}$, $\mathrm{S}=9.5 \mu \mathrm{m}$, respectively. The parameter values of the test structures $\mathrm{A}$ and $\mathrm{B}$ are never used in training. 


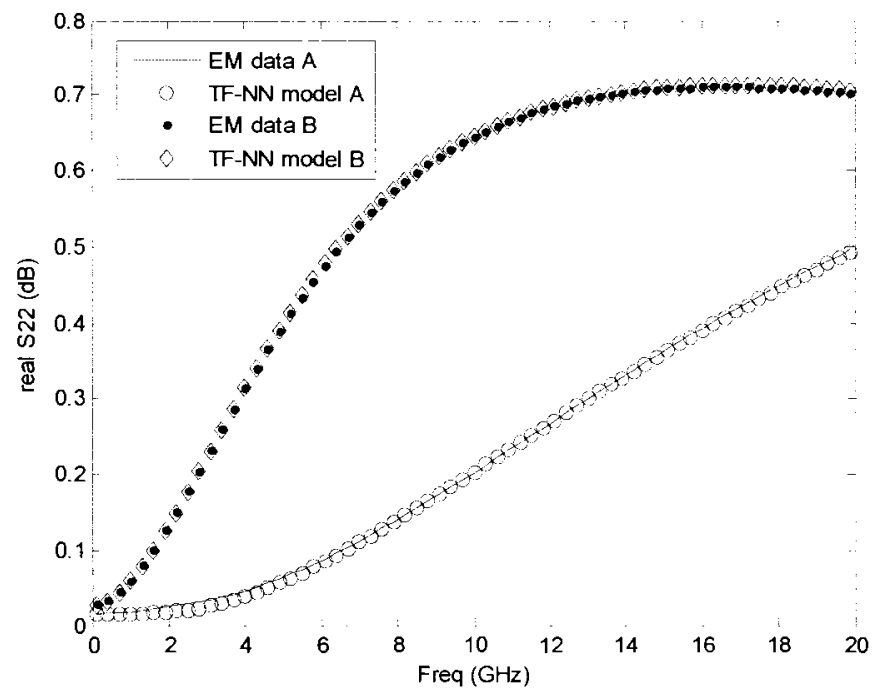

(a)

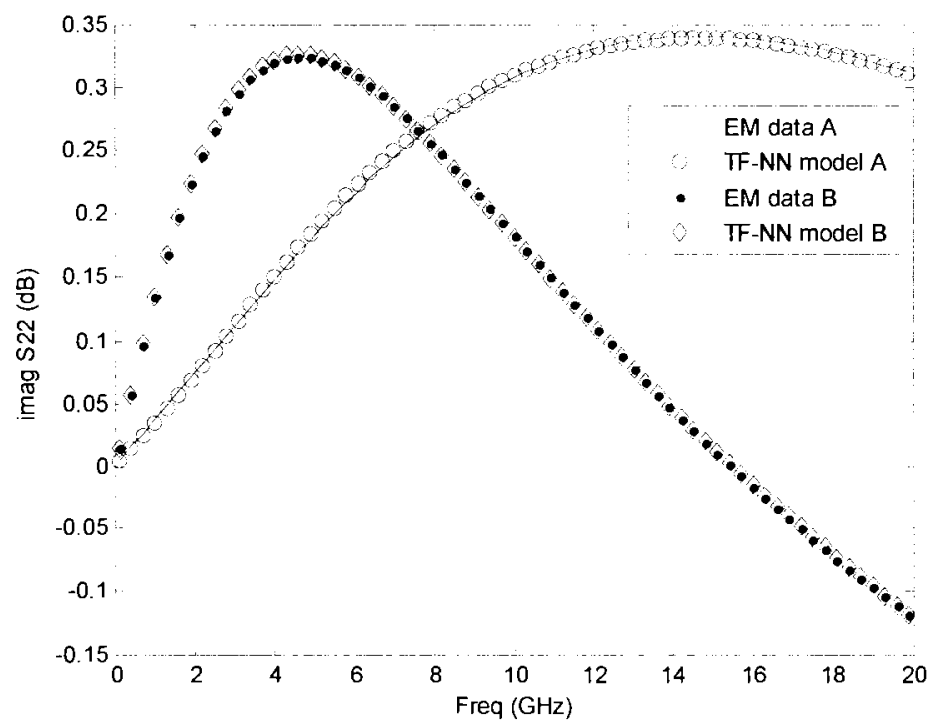

(b)

Figure 3-10. Comparisons of the real and imaginary parts of the S22 for the square-spiral inductor using the TF-NN model and detailed EM simulation, Curve $A$ and Curve $B$ are generated based on $\mathrm{N}=1.5, R_{i n}=33 \mu \mathrm{m}, \mathrm{W}=6 \mu \mathrm{m}, \mathrm{S}=1.5 \mu \mathrm{m}$ and $\mathrm{N}=1.5, R_{i n}=123 \mu \mathrm{m}, \mathrm{W}=6 \mu \mathrm{m}$, $\mathrm{S}=9.5 \mu \mathrm{m}$, respectively. The parameter values of the test structures $\mathrm{A}$ and $\mathrm{B}$ are never used in training. 
Comparison of the CPU time by using the EM simulation and the TF-NN model are shown in Table 3-VII. For each example, the CPU time is calculated based on 30 sets of different structures. The calculation of the CPU time is based on $2.4 \mathrm{GHz}$ Intel Core 2 CPU and $2046 \mathrm{MB}$ RAM. It is observed that the TF-NN model can achieve a competitive accuracy as detailed EM simulation and can be much faster than EM simulator.

Table 3-VII. CPU time comparisons for all three examples by using the EM simulator and the TF-NN model.

\begin{tabular}{|c|c|c|}
\hline & $\begin{array}{c}\text { Simulation time for simulating } \\
\text { all the 30 structures within each } \\
\text { example's training range using } \\
\text { EM simulators }\end{array}$ & $\begin{array}{c}\text { Simulation time for all the 30 } \\
\text { structures within each } \\
\text { example's training range using } \\
\text { TF-NN model }\end{array}$ \\
\hline Example1 & 11 hours & $0.66 \mathrm{~s}$ \\
\hline Example2 & 12.67 hours & $0.71 \mathrm{~s}$ \\
\hline Example 3 & 19 hours & $1.07 \mathrm{~s}$ \\
\hline
\end{tabular}




\subsection{Conclusions}

We have presented an alternative approach for accurate and efficient modeling of the passive microwave components. The embedded transfer function in the TF-NN model provides additional information for components modeling and helps to reduce the complexity of the mapping of the neural networks. The reduction in the mapping complexity also reduces the amount of training data for developing the microwave component models. This TF-NN model can also help to provide fast and accurate solutions without exploring equivalent circuit topology. This method has been applied to via and square inductor modeling. These application examples have demonstrated that the combined TF-NN model is fast and accurate for passive microwave components modeling even if the equivalent circuits are unavailable. This combined TF-NN model is proved to help to speed up the passive microwave component modeling and can be used in high-level microwave circuit design. 


\section{Chapter 4:}

\section{A Neural Network Based Approach to the Design and Optimization of the Microstrip Power Divider}

\subsection{Introduction}

The microstrip power divider is one of the important passive components and widely used in many microwave and millimeter-wave systems and devices such as antenna feeding systems, power amplifiers, and etc. The most popular structure is the Wikinson power divider, which was invented in 1960 [66]. This structure has wide applications in microwave circuits and antenna feeds. However, its narrow bandwidth limits its applications in the modern broadband communication systems. In recent years, several schemes have been devised to increase the bandwidth of the Wikinson power divider [6769]. Although these improved methods can get a much more sufficient design with dualbandwidth now, an optimal design result for the high-frequency and high-speed optimization is still hard to achieve. The available commercial microwave software packages such as Sonnet and HFSS can offer excellent accuracy. However, they are mainly devised for the analysis of the microwave structures. Their applications for the design and synthesis in combination with the optimization routines are very time- 
consuming with respect to machine CPU time and manual effort. Therefore, efficient design and optimization methods for microwave circuits and components are desirable now.

In this chapter, a novel design and optimization procedure based on the neural network technique is proposed to meet the design goal of the microstrip power divider. After the power divider structure is set up, it is first optimized using equivalent circuit to get a good initial structure for the optimization. Getting initial values may be considered as an approximate design procedure of the power divider. The initial power divider structure is then subdivided into several small sub structures. The neural networks are used to learn the EM behaviour of these sub structures. Inputs of the neural sub models are the geometrical/physical parameters of the EM sub structures while the outputs of the models are the corresponding S-parameters of the EM sub structures. After learned the EM data, these neural sub models are combined to form the overall power divider structure. Instead of computing the EM response of the power divider iteratively from the detailed EM simulation, we use the combined neural sub model to provide instant EM response of the power divider during the optimization. The combined neural sub model provides a much more accurate solution than the equivalent circuit and can be much faster than full-wave EM analysis. The CPU time is reduced significantly by using the neural based model.

In Section 4.2, the microstrip power divider design and optimization procedure is presented in details. In section 4.3, a power divider design example using the proposed method is provided to illustrate the validity and efficiency of the proposed design 
approach. The optimization results and the CPU time comparison are also shown in Section 4.3.

\subsection{Proposed Design and Optimization Procedure}

\subsubsection{Problem Statements}

The design purpose is to construct a microstrip power divider from one transmission line with characteristic impedance $Z_{1}$ to 2 identical transmission lines with characteristic impedance $Z_{2}$ in parallel. The goal is to minimize the magnitude of the $\mathrm{S} 11$ from 0 to $f_{\max }$ GHz.

In order to achieve the design goal, we use an EM power divider structure containing $N$ rectangles. The size and position of each rectangle, which are the width of each rectangle, the length of each rectangle and the space between each pair of coupled rectangles, can be varied. The power divider structure is shown in Fig. 4-1. Direct design and optimization using full-wave analysis may take days and slow down the overall design schedule. The equivalent circuit approach is fast. However, because of the EM effects, the equivalent circuit may not be accurate when frequency is high. On the other hand, the power divider structure we used has many discontinuities. It will be difficult to model these discontinuities in equivalent circuit. Therefore, we propose a much more efficient method to improve this situation. 


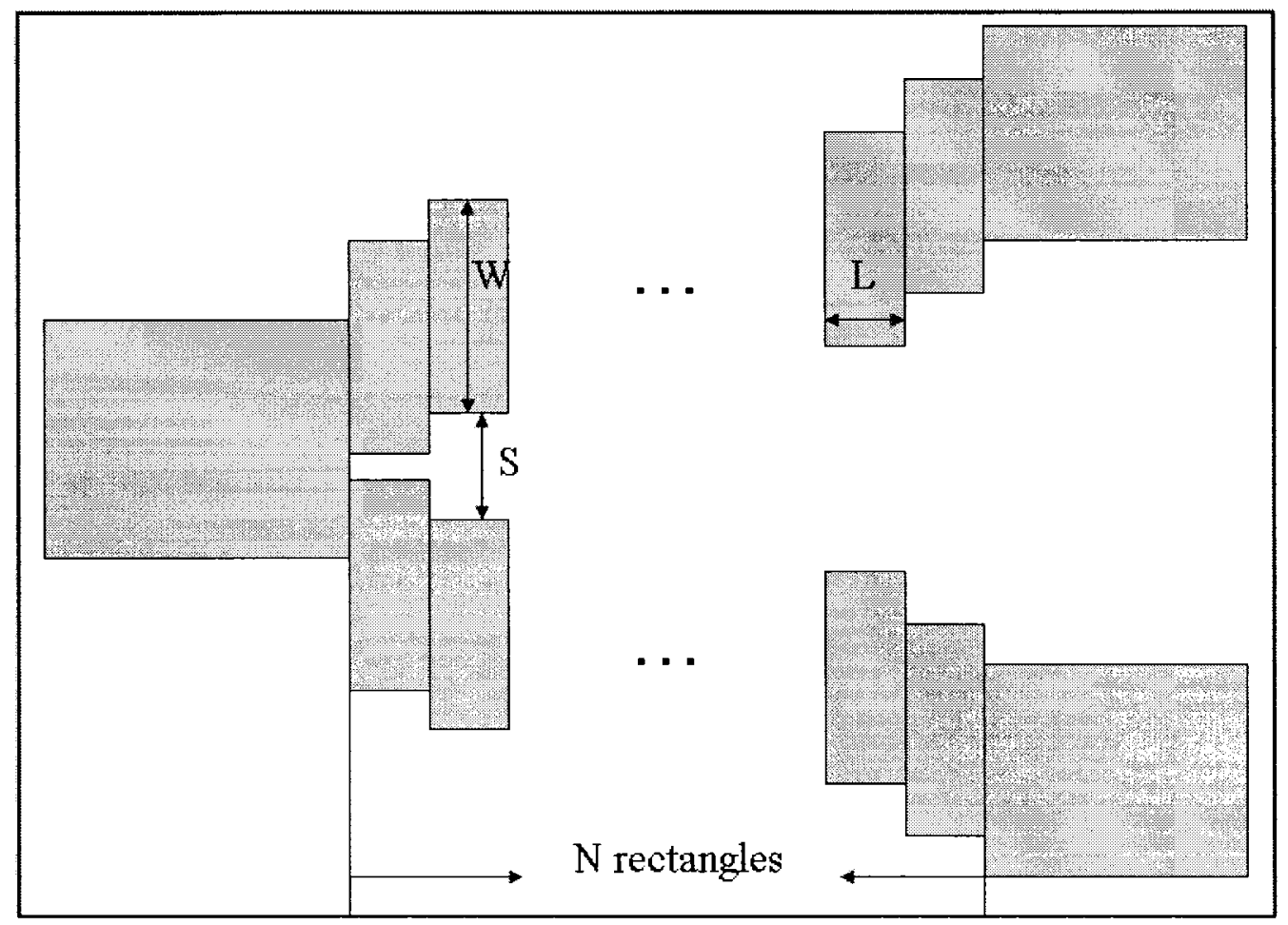

Figure 4-1. The power divider structure with adjustable parameters: W: Width of the rectangle, L: Length of the rectangle, S: Space between each pair of the coupled rectangles.

\subsubsection{Approximate Design Procedure}

The optimal design procedure requires the determination of the initial values of the power divider structure. A good initial point is essential to help to achieve the optimization goal. Therefore, a good initial structure needs to be constructed first. In other words, an approximate design procedure is needed. Although the equivalent circuit of the power divider is not accurate to be based on for design and optimization, it can still give us 
some general idea of the power divider's EM behaviour. Therefore, we use the equivalent circuit at the initial stage of our design to help to find a set of good initial values.

Firstly, an equivalent circuit of the power divider with transmission line is built. This equivalent circuit will be optimized secondly. The geometrical parameter values of the optimized equivalent circuit can be used as the initial values for the power divider optimization.

\subsubsection{Model Subdivision}

Model subdivision is carried out to simplify the EM power divider structure. The most difficult part for the model subdivision is to decide where to split the structure. The criterion when cutting the power divider is keeping a high degree of coupling within an individual sub structure. More circuit subdivision rules can be found in [71]. Illustration of the power divider model subdivision is shown in Fig. 4-2. EM simulator will be used to compute the S-parameters of the sub structures. During the simulation, long transmission lines may need to be added to the sub structure if the sub structure is too short compared to the wavelength. The reason for adding extra transmission lines is if the model itself is too short or the discontinuity is too near the source, local evanescent modes will be created, which may need some distance to decay. The S-parameter definition only makes sense when single dominant mode applies. 


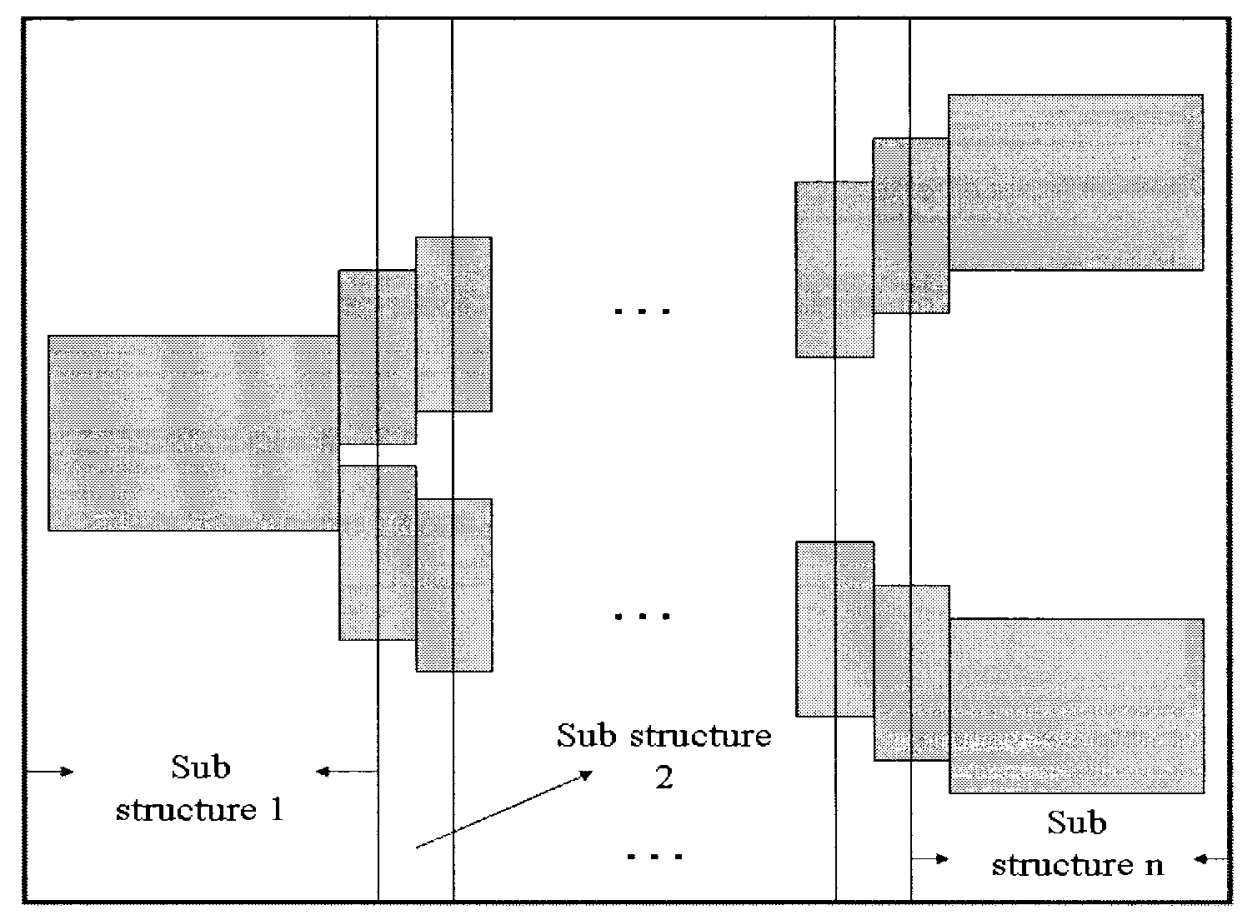

Figure 4-2. Illustration of the subdivision of the EM power divider structure where $n$ is the number of sub structures.

\subsubsection{Neural Network (NN) Model Development}

Let $\boldsymbol{x}$ represents a $N_{\mathrm{x}}$-vector as the neural network inputs. It contains the geometrical parameters of the power divider EM sub structures, e.g., lengths and widths of the rectangles. Let $\boldsymbol{y}$ represent a $N_{\mathrm{y}}$-vector as the neural network outputs, which contains the response of the components under consideration, e.g., S-parameters. The physics/EM relationship between $\boldsymbol{x}$ and $\boldsymbol{y}$ can be highly nonlinear and multi-dimensional. It can be defined as

$$
y=f(x)
$$

Let $\boldsymbol{w}$ be a vector containing all the weight parameters representing the interconnections between inputs and outputs. The definition of $\boldsymbol{w}$ and the manner in 
which $\boldsymbol{y}$ is computed from $\boldsymbol{x}$ and $\boldsymbol{w}$ determine the structure of neural network. Let the neural network model be defined as

$$
\boldsymbol{y}=f_{N N}(\boldsymbol{x}, \boldsymbol{w}) .
$$

MLP structure is used for the neural network structure as shown in Fig. 4-3.

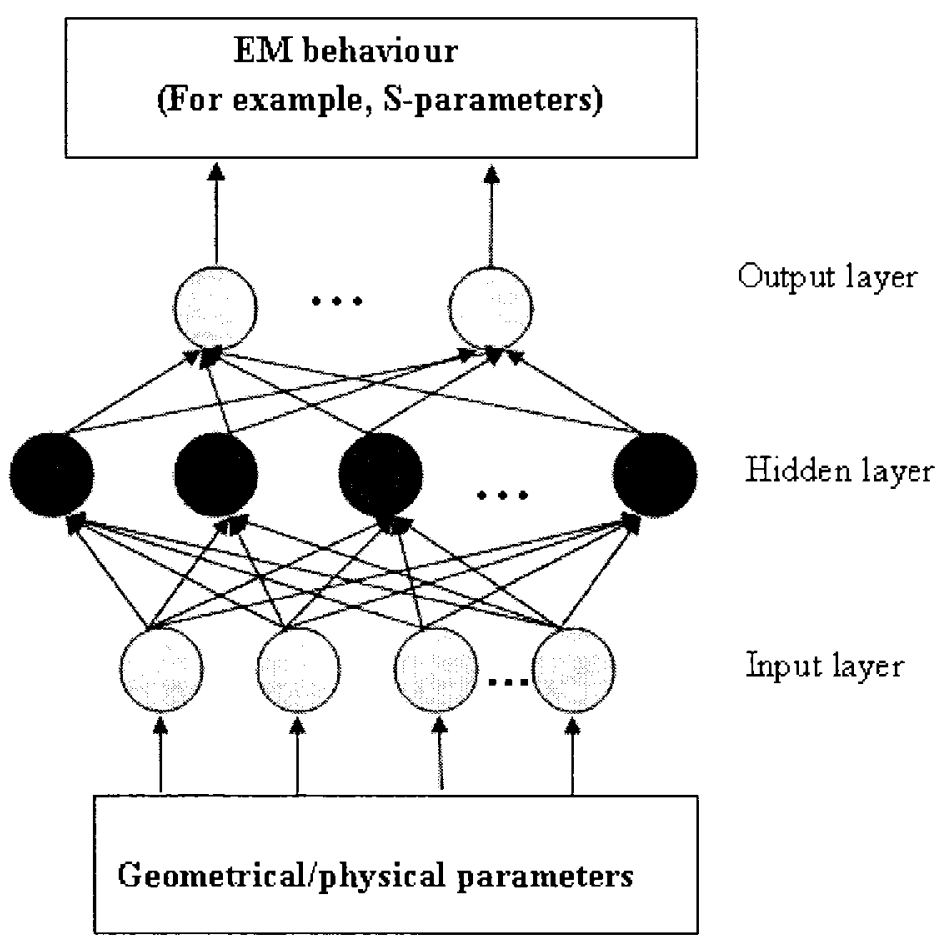

Figure 4-3. The MLP neural network structure used in power divider design.

To generate training data to train the neural networks, we use the EM simulator to obtain the output $y$ from the given values of $x$. Many samples of $(x, y)$ are needed for neural network training. Let $x_{\mathrm{j}}$ be the input sample of $x$. Let $\boldsymbol{d}_{\mathrm{j}}$ be the output sample of $\boldsymbol{y}$. 
The design problem can be described as a nonlinear relationship between $\boldsymbol{x}_{\mathrm{j}}$ and $\boldsymbol{d}_{\mathrm{j} .} \boldsymbol{x}_{\mathrm{j}}$ and $\boldsymbol{d}_{\mathrm{j}}$ can be grouped together as the training data: $\left(\boldsymbol{x}_{\mathrm{j}}, \boldsymbol{d}_{\mathrm{j}}\right)$. Let $T$ be the index set of training data and $j$ be the sample index. By setting $p=2$ in (2.6), the error function during training is defined as

$$
E(\boldsymbol{w})=\frac{1}{2} \sum_{j \in T} \sum_{k=1}^{N_{y}}\left(y_{k}\left(\boldsymbol{x}_{j}, \boldsymbol{w}\right)-d_{j k}\right)^{2}
$$

where $d_{\mathrm{jk}}$ is the $k$ th element of $\boldsymbol{d}_{\mathrm{j}}, y_{\mathrm{k}}\left(\boldsymbol{x}_{\mathrm{j}}, \boldsymbol{w}\right)$ is the $k$ th output of the neural network for the input sample $\boldsymbol{x}_{\mathrm{j}}$. The purpose of training is to adjust $\boldsymbol{w}$ such that the error function $E(\boldsymbol{w})$ is minimized. The neural sub models are trained to learn the EM sub structures' response obtained through the model subdivision and the EM simulation. The training can be done through NeuroModeler [63]. At the end of training, these neural sub models can represent general linear/nonlinear relationships and be used online during the power divider design and optimization stage providing fast model evaluation in replace of the original slow models.

\subsubsection{Power divider Optimization}

After the neural sub models are well trained, they are now ready to be used for the optimization. The trained neural sub models are combined the same way that the power divider model is split. The combined neural sub model is then plugged into NeuroADS [72]. NeuroADS is a plug-in module for Agilent-ADS [73]. Through NeuroADS, the neural models that are built using NeuroModeler can be used by Agilent-ADS for circuit simulation and design. Several iterations of ADS optimization will be performed. The 
number of iterations will be determined based on the optimization goal. A minimized S11 will be achieved through this optimization procedure.

\subsection{Demonstration Example of the Microstrip Power Divider Design and Optimization Using the Proposed Design Method}

\subsubsection{EM Power Divider Structure Development}

In this example, we construct a power divider from one $17.5 \mathrm{ohm}$ transmission line to 2 identical $35 \mathrm{ohm}$ transmission lines in parallel. The broadband match transition goal is from 0 to $30 \mathrm{GHz}$, during which the magnitude of the $\mathrm{S} 11$ is minimized.

The micorstrip power divider's EM structure contains 12 copper rectangles. The size and position of each rectangle can be varied. These variables can be described as the width of each rectangle, the length of each rectangle and the space between each pair of coupled rectangles. Fig. 4-4 shows the 2D power divider structure. The power divider has been designed for a substrate with a relative permittivity 2.9 , loss tangent 0.01 and dielectric thickness 10 mils. The 3D structure of the power divider is shown in Fig. 4-5. 


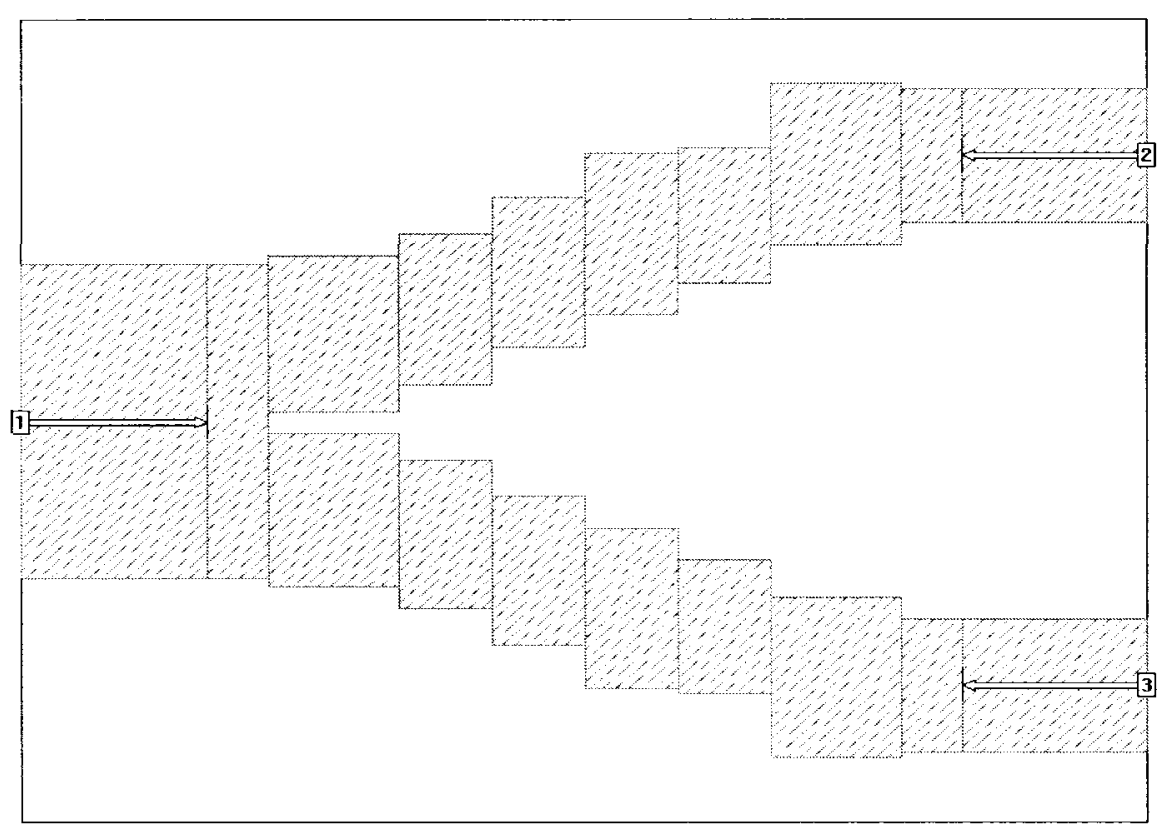

Figure 4-4. Rectangle power divider structure.

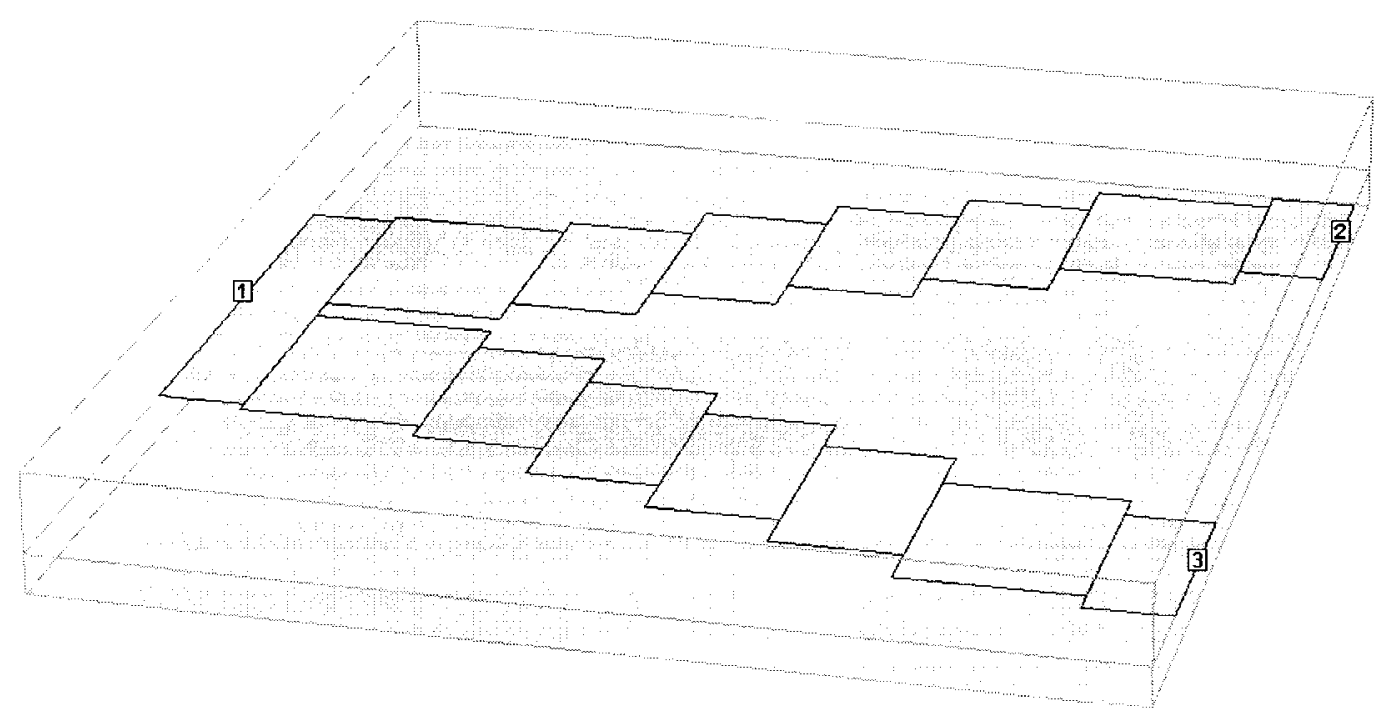

Figure 4-5. 3D view of the power divider structure. 


\subsubsection{Derivation of Initial Values}

Because of the need of good initial values for the optimization procedure, an equivalent circuit of the EM power divider structure is built using Agilent-ADS. The copper rectangles are replaced with transmission lines in Agilent-ADS. The equivalent circuit model is optimized with ADS build-in optimization function. The transmission line power divider structure is shown in Fig. 4-6. The geometrical parameter values of the equivalent circuit can be used as the initial values for the power divider optimization. The initial geometrical parameter values of the power divider are listed in the second column of Table 4-III. The simulation results of the EM structure with initial values are shown in Fig. 4-7. The initial minimum value for $\mathrm{S} 11$ is $-14.2 \mathrm{~dB}$. All the detailed EM simulation results in this chapter are achieved by using the EM simulator Sonnet [65].

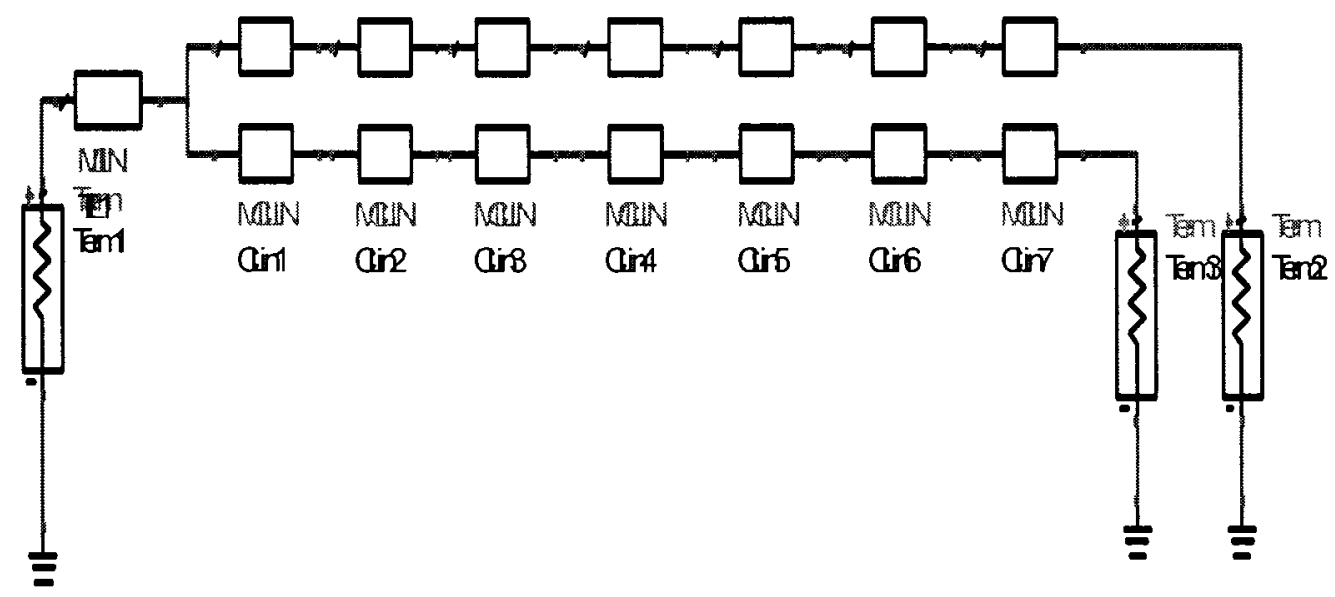

Figure 4-6. Equivalent circuit of the power divider built with Agilent-ADS. 


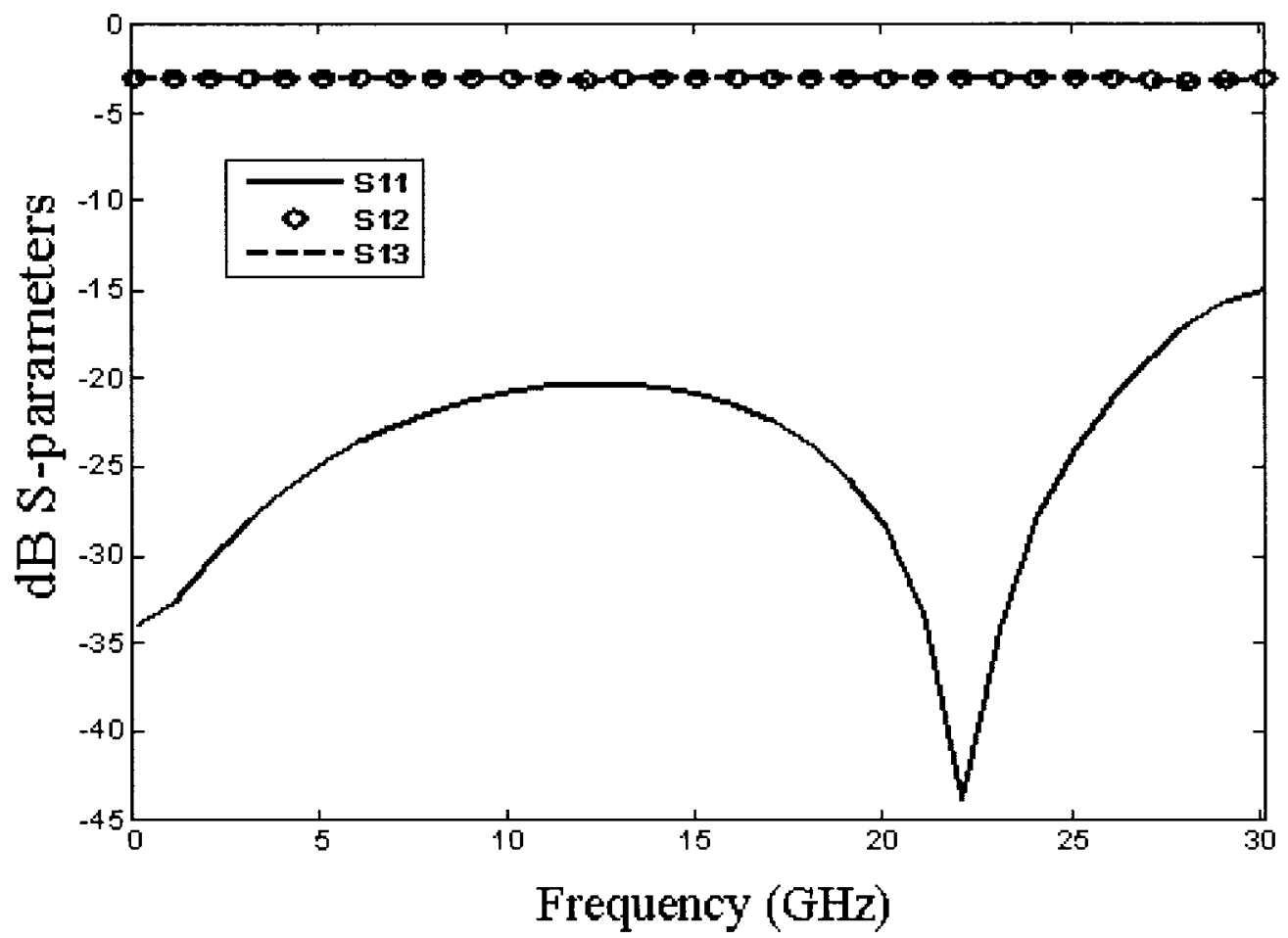

Figure 4-7. EM simulation results of the power divider structure based on initial optimized parameter values: dB S11, dB S12 and dB S13.

\subsubsection{Power Divider Model Subdivision}

Following the model subdivision rules in [71], the power divider structure is subdivided into 14 pieces as shown in Fig. 4-8. These 14 sub structures can be further grouped into four types of structures: 1:2 power splitter structure as shown in Fig. 4-9(a), difference between two coupled rectangles as shown in Fig. 4-9(b) and coupled line structure as shown in Fig. 4-9(c). The last pair of output lines is shown in Fig. 4-9(d). As the output impedance is fixed, the last pair of output lines is fixed, too. A comparison of EM simulation results using overall EM power divider structure and combined EM sub 
structure is shown in Fig. 4-10. These simulation results are based on the power divider's initial parameter values.

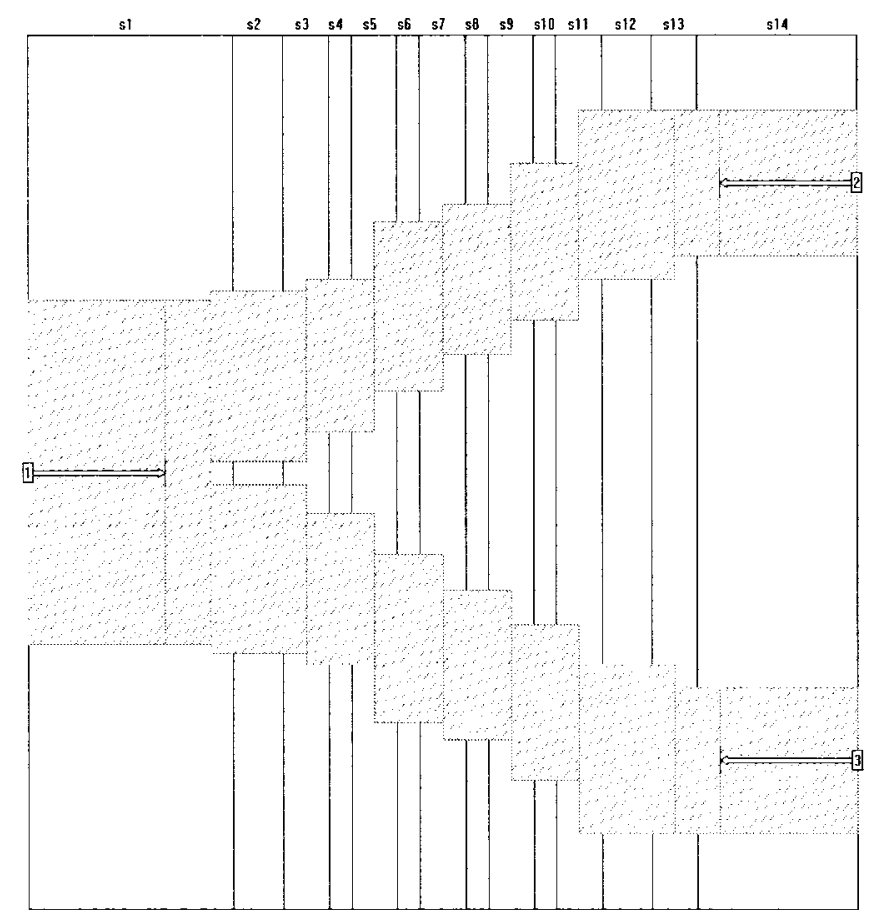

Figure 4-8. 14 sub structures of the overall power divider structure after model subdivision. 


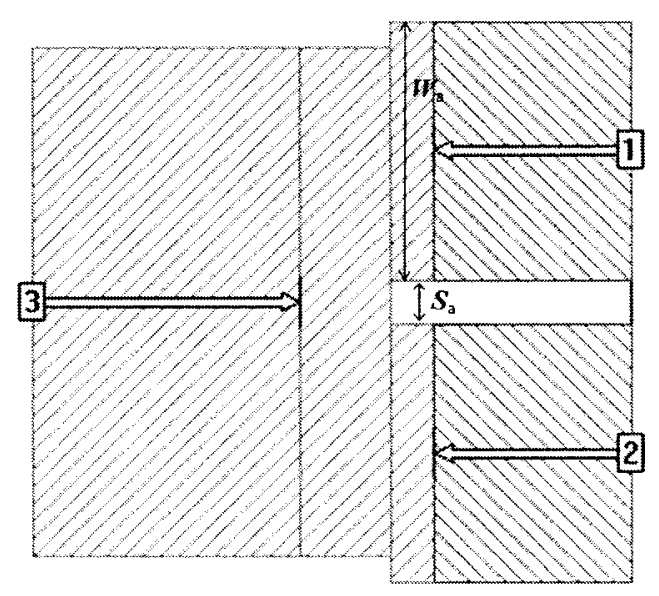

(a)
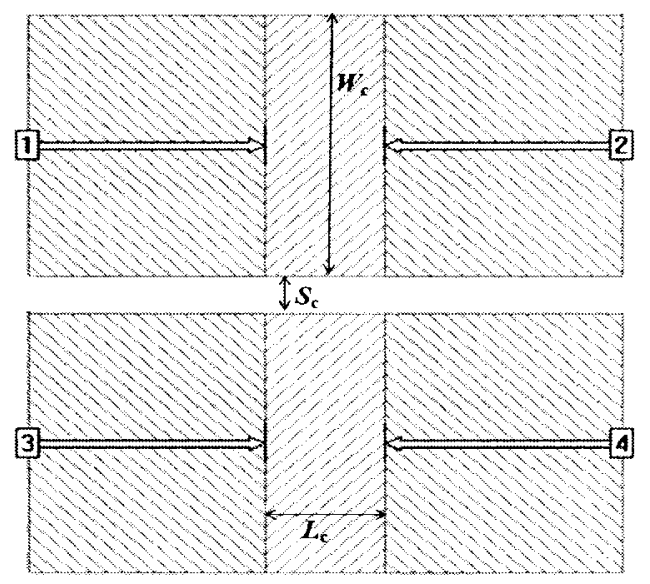

(c)

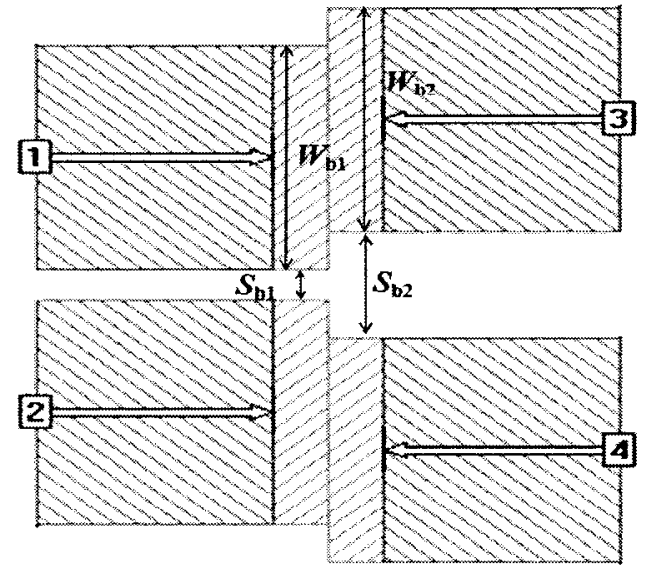

(b)
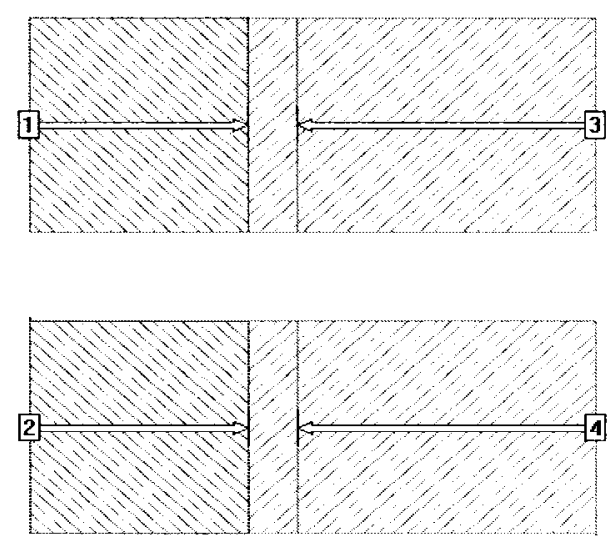

(d)

Figure 4-9. Four types of EM sub structures: (a) 1:2 power splitter, (b) Coupled difference between two rectangles, (c) Coupled lines, (d) Coupled output lines. 


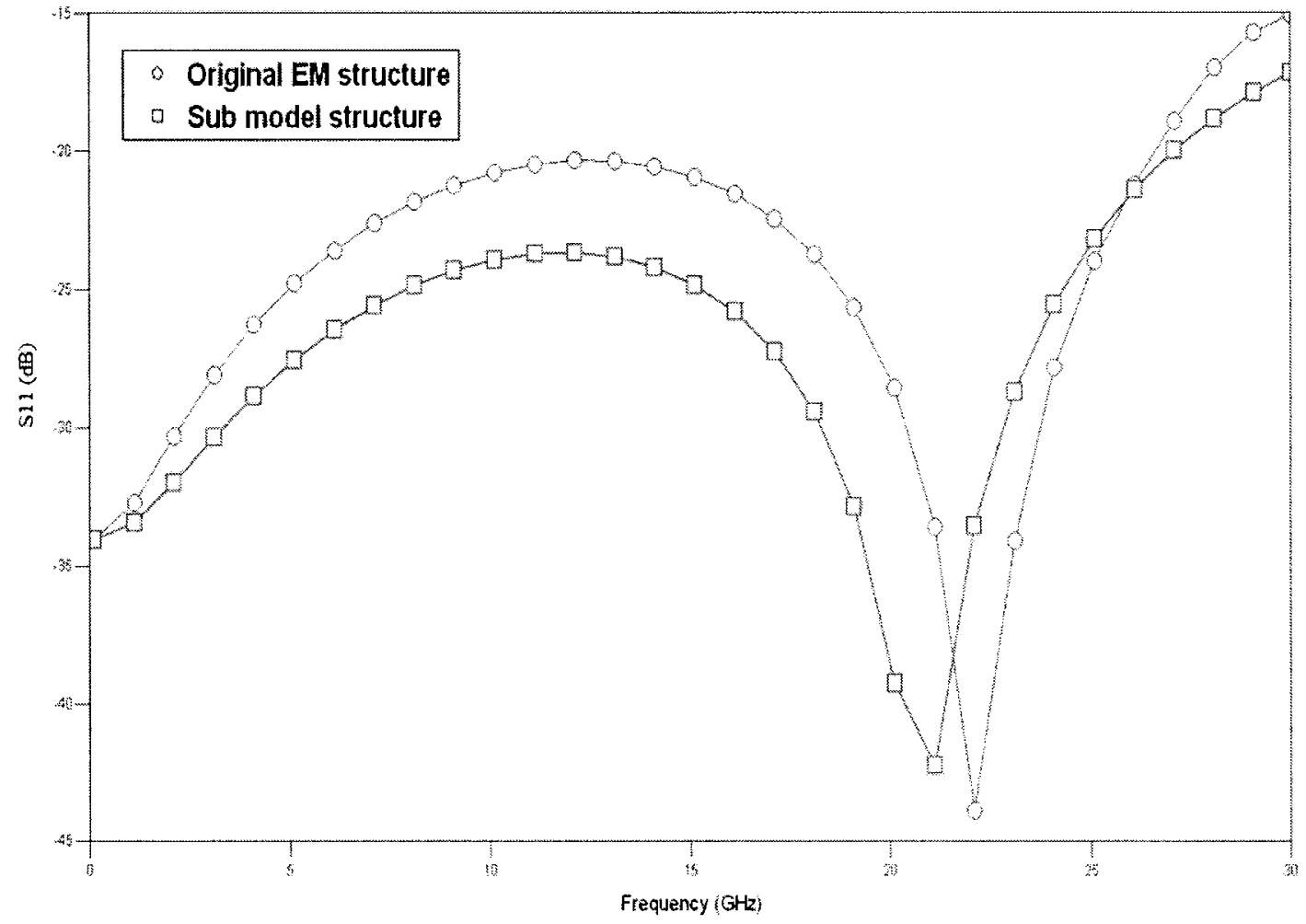

Figure 4-10. A comparison of the EM simulation results using the overall EM structure and the combined EM sub structure: The simulations are based on the power divider's initial parameter values. 


\subsubsection{Neural Network (NN) Models Development and EM Data Generation}

Three neural sub models are needed for the design. The outputs of the three neural models are the corresponding real and imaginary part of S-parameters. The inputs of the first neural sub model are the width of the coupled rectangles $W_{\mathrm{a}}$, the space between the coupled rectangles $S_{\mathrm{a}}$ and the frequency. The geometrical parameters of the first neural sub model are shown in Fig. 4-9(a). The first neural sub model is shown in Fig. 4-11(a). The inputs of the second neural sub model are the width of the first coupled rectangles $W_{\mathrm{b} 1}$, the width of the second coupled rectangles $W_{\mathrm{b} 2}$, the space between the first pair of coupled rectangles $S_{\mathrm{b} 1}$, the space between the second pair of coupled rectangles $S_{\mathrm{b} 2}$ and the frequency. The geometrical parameters of the second neural sub model are shown in Fig. 4-9(b). The second neural sub model is shown in Fig. 4-11(b). The inputs of the third neural sub model are the length of the rectangles $L_{\mathrm{c}}$, the width of the rectangles $W_{\mathrm{c}}$, the space between the coupled rectangles $S_{\mathrm{c}}$ and the frequency. The geometrical parameters of the third neural sub model are shown in Fig. 4-9(c). The third neural sub model is shown in Fig. 4-11(c). 


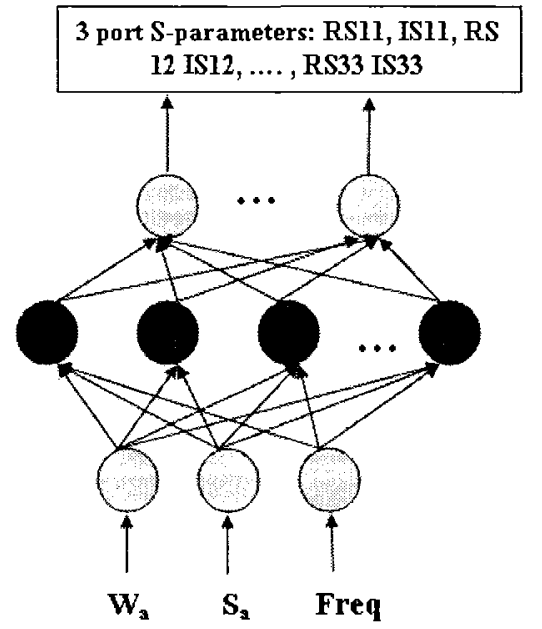

(a)

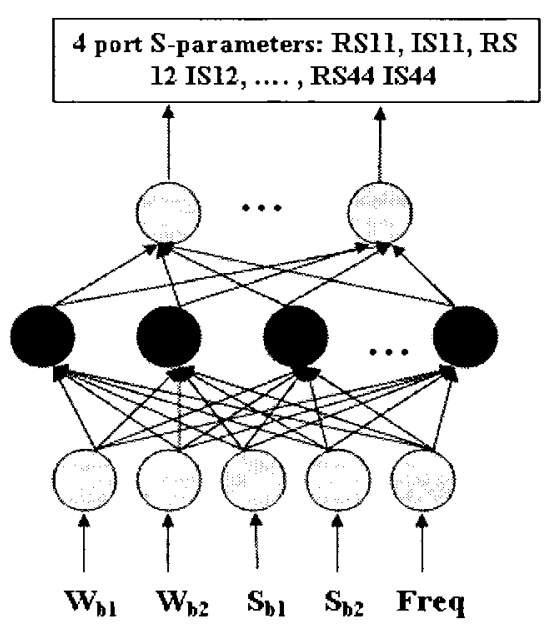

(b)

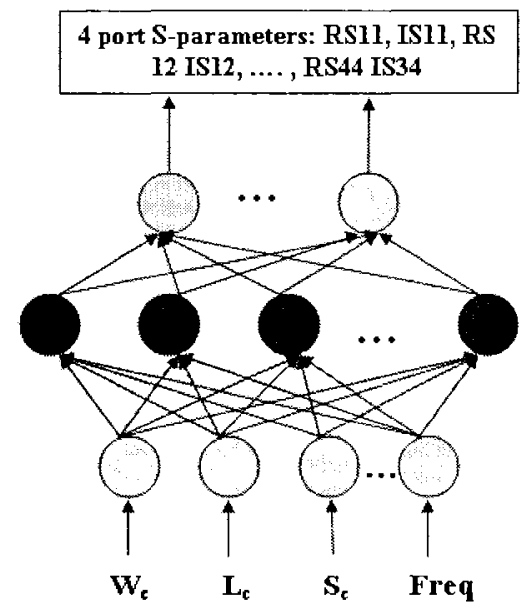

(c)

Figure 4-11. Three neural sub models used for the power divider design and optimization: (a) Neural sub model 1, (b) Neural sub model 2, (c) Neural sub model 3. 
The training for these 3 neural sub models is done through NeuroModeler. The training is based on Quasi-Newton Method. The training errors are listed in Table 4-I. The input ranges of the three neural sub models are listed in Table 4-II. Training data used in training process are from the detailed EM simulation. The required EM data necessitates the repetitive EM simulation for different values of geometrical/physical parameters. The conventional manual operation of EM simulator demands constant human involvement. Therefore, we develop an automation mechanism for the EM data generation based on the NeuroAMG [74] to overcome this challenge. NeuroAMG allows us to implement the EM data generation into a computer-based process. The EM simulator driver is defined once before beginning the data generation. Whenever the geometries change, the driving mechanism automatically updates inputs of the EM simulator and activates EM simulation of the updated geometry.

Table 4-I. Training errors of the three neural sub models for the power divider design.

\begin{tabular}{|c|l|l|}
\hline Model & Average errors (\%) & Worst-Case errors (\%) \\
\hline Neural sub model1 & 0.0212 & 0.3306 \\
\hline Neural sub model2 & 0.023 & 0.3879 \\
\hline Neural sub model3 & 0.0311 & 0.487 \\
\hline
\end{tabular}


Table 4-II. Training ranges of the three neural sub models of the power divider.

\begin{tabular}{|c|c|}
\hline \multicolumn{2}{|c|}{ Neural sub model 1} \\
\hline Input & Range \\
\hline$W_{\mathrm{a}}$ & 50 to $60 \mathrm{mils}$ \\
\hline$S_{\mathrm{a}}$ & 6 to 140 mils \\
\hline Frequency & 0 to $30 \mathrm{GHz}$ \\
\hline \multicolumn{2}{|c|}{ Neural sub model 2} \\
\hline Input & Range \\
\hline$W_{\mathrm{b} 1}$ & 50 to $60 \mathrm{mils}$ \\
\hline$W_{\mathrm{b} 2}$ & 50 to $60 \mathrm{mils}$ \\
\hline$S_{\mathrm{b} 1}$ & 6 to 140 mils \\
\hline$S_{\mathrm{b} 2}$ & 6 to 140 mils \\
\hline Frequency & 0 to $30 \mathrm{GHz}$ \\
\hline \multicolumn{2}{|c|}{ Neural sub model 3} \\
\hline Input & Range \\
\hline$L_{\mathrm{c}}$ & 10 to $22 \mathrm{mils}$ \\
\hline$W_{\mathrm{c}}$ & 50 to 60 mils \\
\hline$S_{\mathrm{c}}$ & 6 to 140 mils \\
\hline Frequency & 0 to $30 \mathrm{GHz}$ \\
\hline
\end{tabular}




\subsubsection{Combined Neural Sub Model Development and Optimization Results}

After the neural sub models are trained, they are plugged into NeuroADS to be combined to form the overall EM power divider structure. The combined neural sub model is shown in Fig. 4-13. We then test the combined neural sub model to check the validity of the model. During the test, the combined neural sub model is compared with the EM data of the combined EM sub structure. The test results for two sets of different test data are shown in Fig. 4-12. The test data are within the training data range but never used in training. The geometrical parameter values for test structure 1 are listed in the third column of the Table 4-III. The geometrical parameter values for test structure 2 are listed in the fourth column of the Table 4-III.

From Fig. 4-12 we can see that, the combined neural sub model is very consistent with the EM data of the combined EM sub structure. Therefore, the combined neural sub model is ready to be used in place of the detailed EM simulation for the optimization. The ADS optimization is performed and 75 iterations are used. A much smaller S11 is achieved through ADS optimization. The optimized power divider geometrical parameter values are listed in the fifth column of Table 4-III. A comparison of EM simulation results of the initial power divider structure and the optimized power divider structure are shown in Fig. 4-14. The optimized S11 is $-23.3 \mathrm{~dB}$ as shown by the horizontal line in Fig. 4-14. 


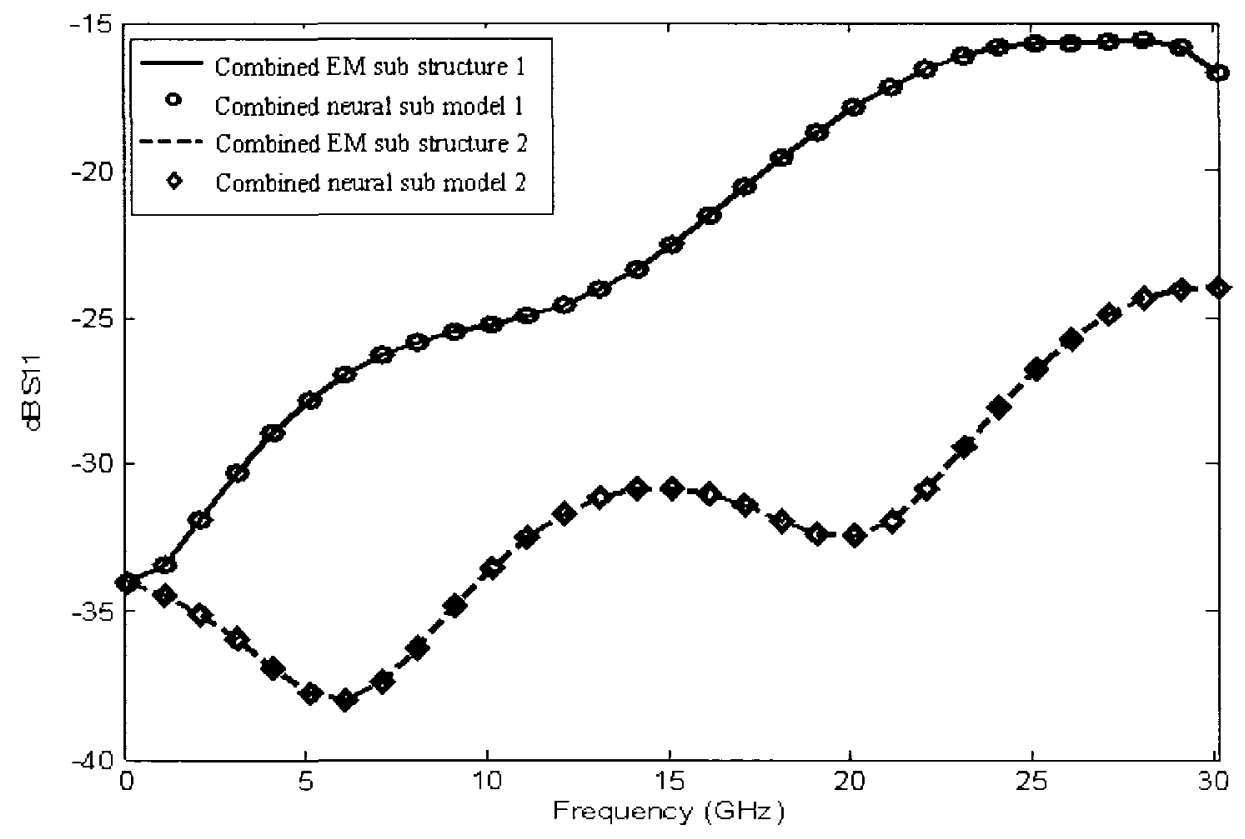

Figure 4-12. Comparisons of simulation results using combined EM sub structure and combined neural sub model. 2 different power divider structures are used, which are listed in the third and fourth column of Table 4-III. 


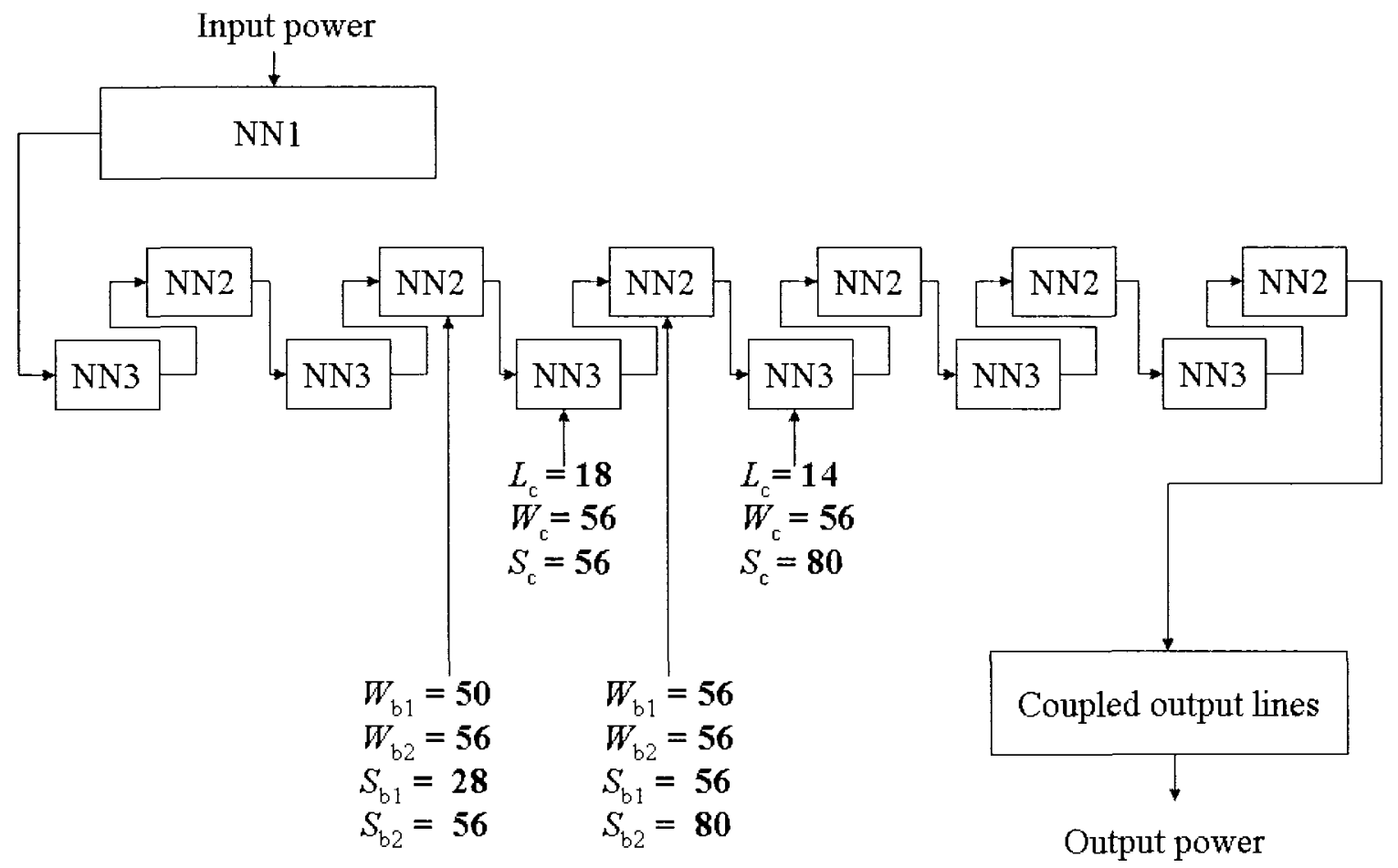

Figure 4-13. Combined neural sub model for the power divider optimization. 


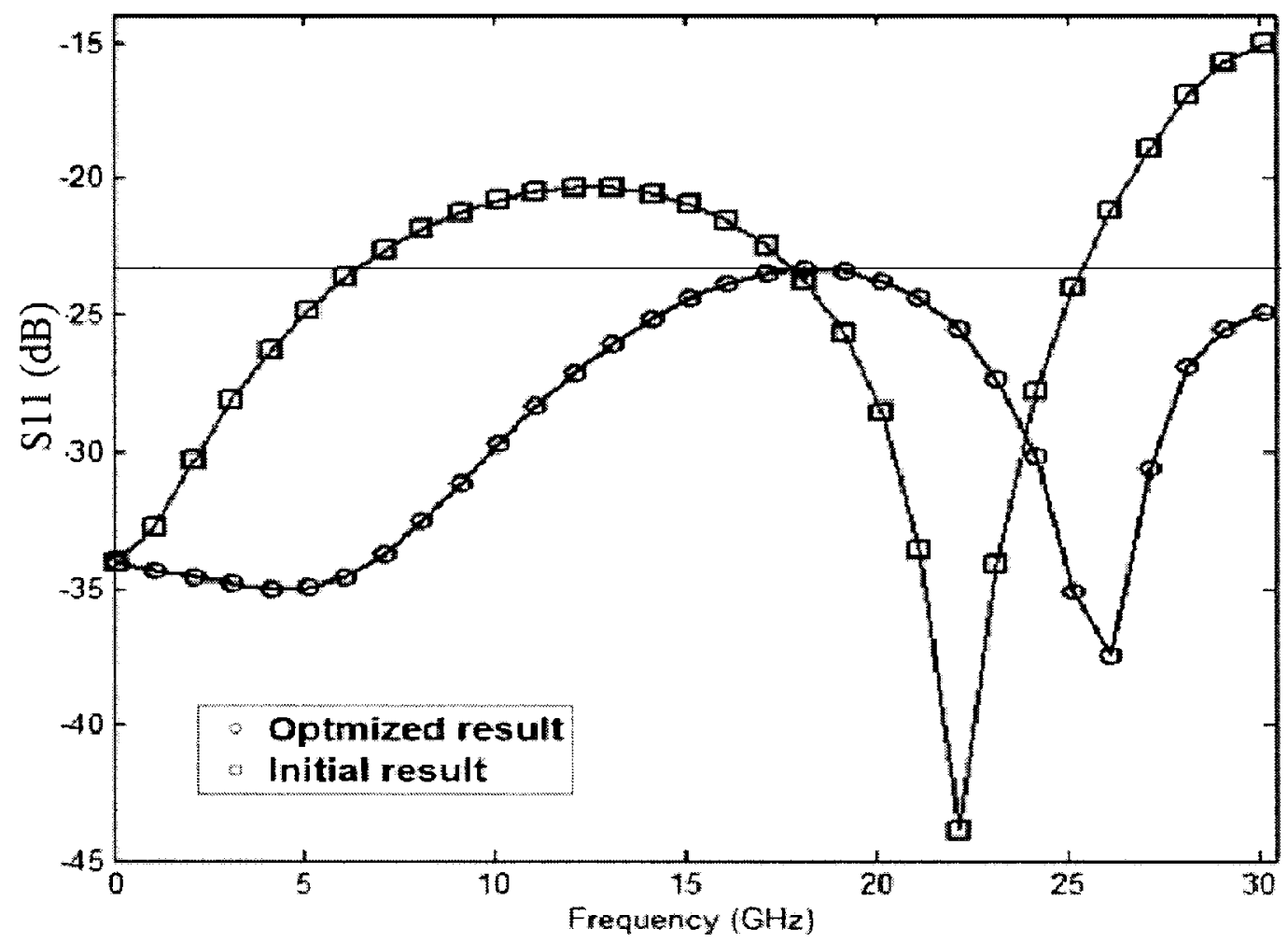

Figure 4-14. A comparison of the optimized S11 and the initial S11 of the power divider.

A comparison of the power divider simulation results computed using the overall EM structure, the equivalent circuit, the combined EM sub structure and the combined neural sub model are shown in Fig. 4-15. These simulations are based on the optimized structure parameter values achieved by using the proposed method. It is clearly shown in Fig. 4-15 that the combined neural sub model based design is much more accurate than the conventional equivalent circuit based design over a broad frequency range. Therefore, the 
neural model based optimization gives us a better minimized S11 compared to the initial equivalent circuit based optimization.

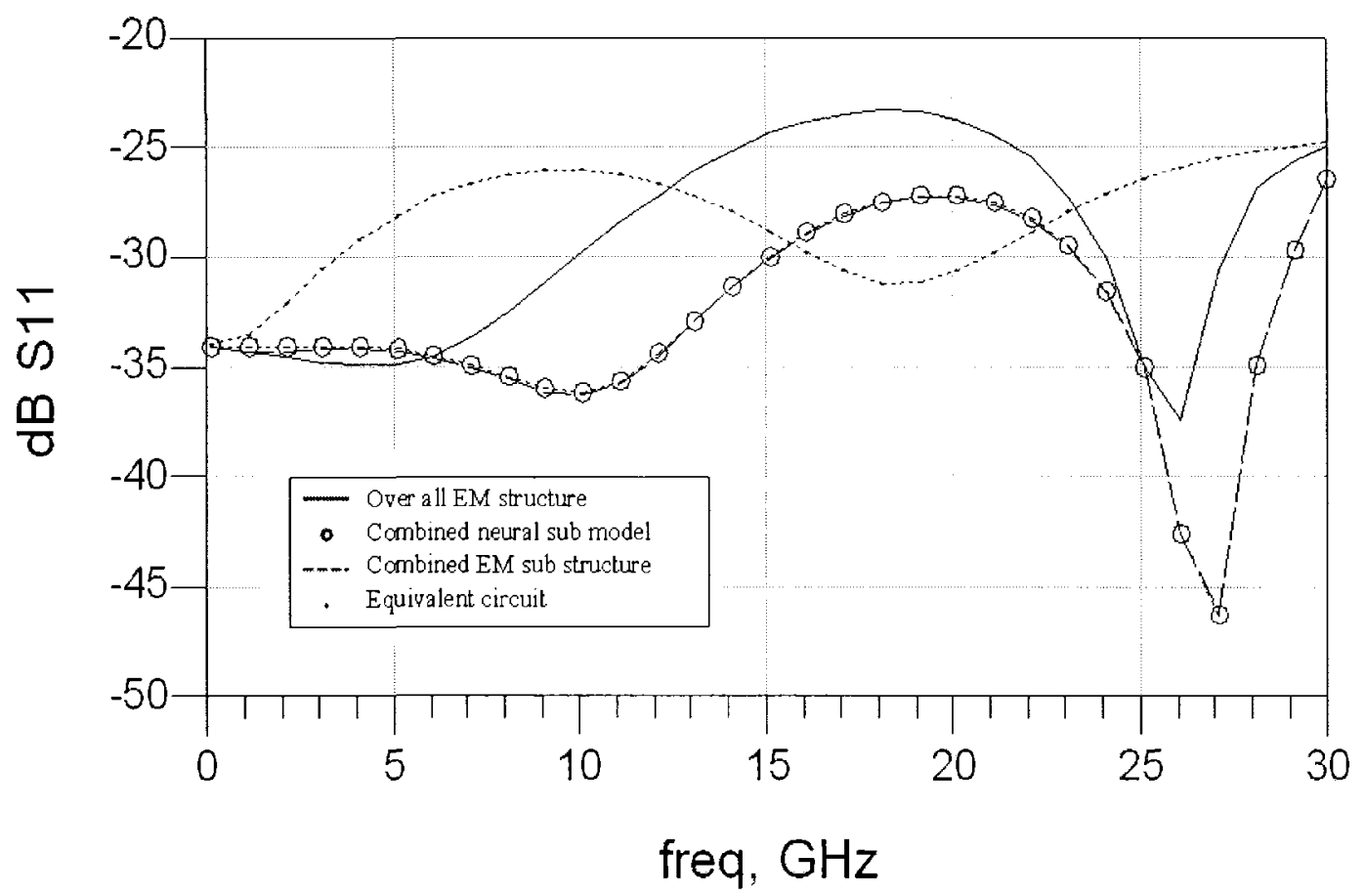

Figure 4-15. The comparison of the power divider simulation results computed using the combined neural sub model, the combined EM sub structure, the equivalent circuit and the overall EM structure. The structure parameter values for all these simulations are the optimized ones achieved by using the proposed method. 
Table 4-III. Geometrical parameter values used in the power divider design and optimization example.

\begin{tabular}{|c|c|c|c|c|}
\hline & $\begin{array}{c}\text { Geometrical values } \\
\text { of the initial } \\
\text { power divider } \\
\text { structure } \\
\text { (mils) }\end{array}$ & $\begin{array}{c}\text { Test geometrical } \\
\text { values } \\
\text { of the } \\
\text { combined neural } \\
\text { sub model } 1 \\
\text { (mils) }\end{array}$ & $\begin{array}{c}\text { Test geometrical } \\
\text { values } \\
\text { of the } \\
\text { combined neural } \\
\text { sub model } 2 \\
\text { (mils) }\end{array}$ & $\begin{array}{c}\text { Geometrical } \\
\text { values } \\
\text { of the optimized } \\
\text { power divider } \\
\text { structure } \\
\text { (mils) }\end{array}$ \\
\hline W1 & 53 & 58 & 50 & 58 \\
\hline W2 & 52 & 56 & 50 & 52 \\
\hline w3 & 52 & 56 & 56 & 58 \\
\hline W4 & 49 & 56 & 56 & 52 \\
\hline W5 & 52 & 54 & 54 & 54 \\
\hline$\overline{\mathrm{W} 6}$ & 51 & 52 & 52 & 58 \\
\hline L1 & 42 & 40 & 38 & 42 \\
\hline $\mathbf{L 2}$ & 30 & 32 & 34 & 30 \\
\hline L3 & 28 & 32 & 34 & 30 \\
\hline $\mathbf{L 4}$ & 22 & 32 & 34 & 30 \\
\hline L5 & 34 & 32 & 34 & 30 \\
\hline L6 & 51 & 40 & 38 & 42 \\
\hline S1 & 12 & 8 & 6 & 8 \\
\hline S2 & 22 & 28 & 28 & 28 \\
\hline $\mathbf{S 3}$ & 66 & 56 & 56 & 56 \\
\hline S4 & 90 & 80 & 80 & 80 \\
\hline S5 & 94 & 104 & 104 & 104 \\
\hline S6 & 140 & 132 & 132 & 132 \\
\hline
\end{tabular}


A comparison of CPU time is shown in Table 4-IV. Compared to the detailed EM simulation based optimization, the proposed neural network approach is very efficient. It helps to speed up the microwave power divider's optimization procedure considerably.

Table 4-IV. Comparison of the CPU time for the power divider design and optimization using different simulation methods.

\begin{tabular}{|l|l|l|l|}
\hline & $\begin{array}{l}\text { Simulation using } \\
\text { Equivalent circuit }\end{array}$ & $\begin{array}{l}\text { Simulation using } \\
\text { Full-wave EM } \\
\text { simulator }\end{array}$ & $\begin{array}{l}\text { Simulation using } \\
\text { combined neural } \\
\text { sub model }\end{array}$ \\
\hline $\mathbf{1}$ power divider structure & $7.23 \mathrm{~s}$ & $25 \mathrm{~min}$ & $7 \mathrm{~s}$ \\
\hline 75 power divider structures & $9.3 \mathrm{~min}$ & $20.8 \mathrm{~h}$ & $8.75 \mathrm{~min}$ \\
\hline
\end{tabular}




\subsection{Conclusions}

A new approach for the design and optimization of the microstrip power divider has been presented. The design procedure consists of three critical steps which are the model subdivision, the neural network development and the model optimization. The model subdivision helps to simplify the EM structure and therefore reduces the simulation time. The neural based model obtained by learning the EM behaviour of the power divider's EM sub structures helps to speed up the optimization procedure compared to the detailed EM simulation based optimization. The microwave power divider design and optimization example has demonstrated that the neural network based method can be used in place of CPU-intensive detailed EM design to significantly speed up the design while providing a better accuracy than the equivalent circuit approach. 


\section{Chapter 5:}

\section{Conclusions and Future Work}

\subsection{Conclusions}

This thesis has presented the neural network based techniques for microwave component modeling and design. Two approaches have been discussed to improve the performance of existing RF/microwave circuit modeling and design methods.

The TF-NN model retains the EM level accuracy and can be much faster than the detailed EM simulation. The TF-NN model uses the transfer function to provide additional knowledge for the target problem and therefore reduces the training data during the model development. Another advantage of The TF-NN model is that it can be used in speeding up the RF/microwave component modeling even if an equivalent circuit is unavailable. Application examples have demonstrated that the combined TF-NN model is accurate for microwave passive component modeling, such as via modeling and square inductor modeling. Therefore, the TF-NN model can be used in place of the slow models in high-level microwave circuit design.

We have also presented another approach for microstrip power divider design and optimization. During the design process, the power divider EM structure is first 
simplified by using the model segmentation. The neural models are first used to learn the EM sub structures' behaviour and then combined in a sequence to form the overall structure. Model optimization has been significantly speeded up by using the combined neural sub model. The novel neural network approach to the design and optimization of the microstrip power divider is proved to improve the effectiveness of EM field solvers in EM-based design automation.

\subsection{Suggestions for Future Research}

As the neural networks have the ability of modeling general input-output relationships, they are very effective in representing diverse types of problems. Therefore, the neural network will continue to be an important area of research and help to shape the design automation methodology for high-speed and high-frequency microwave circuits.

Within the thesis's topic, more complicated models can be built to further demonstrate the advantage of the TF-NN model. The more complicated the model, the more obvious the advantage of the TF-NN model is. TF-NN model can also be applied to modeling sub structures in power divider as geometry of the power divider becomes more complex. You may notice that the TF-NN method has only been explored in the frequency domain modeling. Actually, compared to equivalent circuit, the transfer function can be easier to be applied to time domain analysis because of the use of coefficients. Therefore, time domain analysis based on TF-NN method will be an interesting area to step into in the future.

As further requirements for $\mathrm{RF} /$ microwave circuit design will be raised to meet today's rapid development of commercial wireless communication market, efforts in 
creating new complex model will continue to be made. Therefore, developing more efficient neural network based modeling methods will definitely benefit all levels of the $\mathrm{RF} /$ microwave design including modeling, simulation and optimization.

Knowledge based neural network will continue to be the main direction of future research. Different ways of incorporating knowledge into neural networks can be developed to further reduce the training data while keep the EM level of accuracy over a larger input-space range. 


\section{Bibliography}

[1] S. N. Balakrishnan, R. D. Weil, "Neurocontrol: A Literature Survey," Mathematical and Computer Modeling, vol. 23, No. 1-2, pp. 101-117, 1996.

[2] B. S. Cooper, "Selected applications of neural networks in telecommunication systems," Australian Telecommunication Research, vol. 28, No. 2, pp. 9-29, 1994.

[3] T. Alvager, T. J. Smith, and F. Vijai, "The use of artificial neural networks in biomedical technologies: an introduction," Biomedical Instrumentation and Technology, vol. 28, No. 4, pp. 315-322, 1994.

[4] K. Goita, et al, "Literature review of artificial neural networks and knowledge Based systems for image analysis and interpretation of data in remote sensing," Canadian Journal of Electrical and Computer Engineering, vol. 19, No. 2, pp. 53-61, 1994.

[5] Y. G. Smetanin, "Neural networks as systems for pattern recognition: A review," Pattern Recognition and Image Analysis, vol. 5, No. 2, pp. 254-293, 1995.

[6] Nunmaker, J. F. Jr., and R. H. Sprague Jr., "Applications of neural networks in manufacturing," Proceedings of the Twenty-ninth Hawaii International Conference on System Sciences, vol. 2, pp. 447-453, 1996.

[7] Q. J. Zhang, G. L. Creech (Guest Editors), International Journal of RF and Microwave Computer-Aided Engineering, Special Issue on Applications of Artificial Neural Networks to RF and Microwave Design, vol. 9, NY: Wiley, 1999. 
[8] M. Vai, S. Prasad, "Automatic impedance matching with a neural network," IEEE Microwave and Guided Wave Letters, vol. 3, No. 10, pp. 353-354, Oct. 1993.

[9] T. Horng, C. Wang, and N. G. Alexopoulos, "Microstrip circuit design using neural networks," MTT-S Int. Microwave Symp. Dig., pp. 413-416, 1993.

[10] A. H. Zaabab, Q. J. Zhang, and M. Nakhla, "Analysis and optimization of microwave circuits and devices using neural network models," MTT-S Int. Microwave Symp. Dig., pp. 393-396, 1994.

[11] V. B. Litovski, et al, "MOS transistor modeling using neural network," Electronics Letters, vol. 28, No. 18, pp. 1766-1768, 1992.

[12] F. Gunes, F. Gurgen, and H. Torpi, "Signal-noise neural network model for active microwave devices," IEE Proc.-Circuits, Devices, Syst., vol. 143, No. 1, pp. 1-8, Feb. 1996.

[13] K. Shirakawa, et. Al., "A large-signal characterization of an HEMT using a multilayered neural network," IEEE Trans. on Microwave Theory and Techniques, vol. 45, No. 9, pp. 1630-1633, Sept. 1997.

[14] P. M. Watson, and K. C. Gupta, "EM-ANN models for microstrip vias and interconnects in multilayer circuits," IEEE Trans. Microwave Theory and Techniques, vol. 44, pp. 2495-2503, Dec. 1996.

[15] G. L. Creech, et al., "Artificial neural networks for fast and accurate EM-CAD of microwave circuits," IEEE Trans. Microwave Theory and Techniques, vol. 45, pp. 794-802, May. 1997. 
[16] A. Veluswami, M. S. Nakhla, and Q. J. Zhang, "The application of neural networks to EM-based simulation and optimization of interconnects in high-speed VLSI circuits," IEEE Trans. on Microwave Theory and Techniques, vol. 45, pp. 712-723, May 1997.

[17] G. L. Creech, et al, "Artificial neural networks for accurate microwave CAD applications," IEEE MTT-S Int. Microwave Symp. Dig., pp. 733-736, 1996.

[18] P. Watson, and K. C. Gupta, "EM-ANN Models for Via Interconnects in Microstrip Circuits," IEEE MTT-S Int. Microwave Symp. Dig., pp. 1819-1822, 1996.

[19] P. M. Watson, C. Cho, and K. C. Gupta, "EM-ANN model synthesis of physical dimensions for multilayer asymmetric coupled transmission line structures," International Journal of RF and Microwave Computer-Aided Engineering, vol. 9, NO. 3, pp. 175-186, 1999.

[20] P. M. Watson, and K. C. Gupta, "EM-NN modeling and optimal chamfering of $90^{\circ} \mathrm{CPW}$ bends with air-bridges," IEEE MTT-S Int. Microwave Symp. Dig., pp. 2603-1606, June. 1997.

[21] P. M. Watson, and K. C. Gupta, "Design and optimization of CPW circuits using EM-ANN models for CPW connections," IEEE Trans. on Microwave Theory and Techniques, vol. 45, No. 12, pp. 2515-2523, Dec. 1997.

[22] K. C. Gupta, and P. M. Watson, "Transmission line model for CPW antennas using ANN modeling approach, "IEEE AP-S and URSI Radio Science Meeting Digest, Montreal, Canada, pp. 212, July. 1997. 
[23] P. M. Watson, K. C. Gupta, and R. L. Mahajan, "Development of Knowledge Based Artificial Neural Network Models for Microwave Components," IEEE MTT-S Int. Microwave Symp. Dig., pp. 9.12, 1998.

[24] Q. J. Zhang, et al, "Ultra fast neural models for analysis of electro/opto interconnects," IEEE Electronic Components and Technology Conf., San Jose, CA, pp. 1134-1137, May. 1997.

[25] A. E. Smith, and A. k. Mason, "Cost estimation predictive modeling: Regression versus neural network," Engineering Economist, vol. 42, No. 2, pp. 137-160, Feb. 1997.

[26] G. R. Finnie, G. E. Wittig, and J. M. Desharnais, "A comparison of software effort estimation techniques using function points with neural networks, case-based reasoning, and regression models," Journal of Systems an Software, vol. 39, No. 3, pp. 281-290, Mar. 1997.

[27] D. Tsintikidis, et al, "A neural network approach to estimating rainfall from spaceborne microwave data," IEEE Trans. on Geoscience and Remote Sensing, vol. 35, No. 5, pp. 1079-1093, May. 1997.

[28] A. Hierlemann, et al, "Environmental chemical sensing using quartz microbalance sensor arrays: Applications of multicomponent analysis techniques," Sensors and Materials, vol. 7, No. 3, pp. 179-189, Mar. 1995.

[29] Y. Danon, and M. J. EMbrechts, "Least squares fitting using artificial neural networks," in Intelligent Engineering Systems through Artificial Neural Networks, vol. 2, NY: ASME Press, 1992. 
[30] Q. J. Zhang, F. Wang, and M. S. Nakhla, "Optimization of high-speed VLSI interconnects: A review," Int. J. of Microwave and Millimeter-Wave CAE, vol. 7, pp. 83-107, 1997.

[31] F. Wang and Q. J. Zhang, "Knowledge-based neural models for microwave design," IEEE Trans. Microwave Theory Tech., vol. 45, pp. 2333-2343, 1997.

[32] P. M. Watson, K. C. Gupta and R. L. Mahajan, "Application of knowledge-based artificial neural network modeling to microwave components," Int. J. RF and Microwave CAE, vol. 9, No. 3, pp. 254-260, 1999.

[33] J. W. Bandler, M. A. Ismail, J. E. Rayas-Sánchez, and Q. J. Zhang, "Neuromodeling of microwave circuits exploiting space mapping technology," IEEE Trans. Microwave Theory Tech, vol. 47, pp. 2417-2427, 1999.

[34] F. Wang, V. K. Devabhaktuni, C. xi, and Q. J. Zhang, "Neural network structures and training algorithms for $\mathrm{RF}$ and microwave applications," Int. J. RF and Microwave CAE, vol. 9, pp. 216-240, May. 1999.

[35] K. C. Gupta, "Emerging trends in millimeter-wave CAD," IEEE Trans. Microwave Theory and Techniques, vol. MTT-46, pp. 747-755, June. 1998.

[36] T. Itoh, ed., Numerical Techniques for Microwave and Millimetre-Wave Passive Structures, New York: John Wiley and Sons, 1989.

[37] M. N. O., Sadiku, Numerical Techniques in Electromagnetics, Boca Raton, FL: CRC Press, 1992.

[38] A. Taflove, Computational Electrodynamics: The Finite-Difference Time-Domain Method, Norwood MA: Artech House, 1995. 
[39] A. H. Zaabab, Q. J. Zhang and M. S. Nakhla, "A neural network approach to circuit optimization and statistical design," IEEE Trans. Microwave Theory Tech, vol. 43, pp. 1349-1358, 1995.

[40] V. K. Devabhaktuni, C. Xi and Q. J. Zhang, "A neural network approach to the modeling of heterjunction bipolar transistors from S-parameter data," European Microwave Conf., Amsterdam, Netherlands, pp. 306-311, 1998.

[41] K. Shirakawa, M. Shimizu, N. Okubo and Y. Daido, "Structural determination of multilayered large signal neural network HEMT model," IEEE Trans. Microwave Theory Tech., vol. 46, pp. 1367-1375, 1998.

[42] P. Burrascano, M. Mongiardo, C. Fancelli, "A neural network model for CAD and optimization of microwave filters," IEEE Int. Microwave Symp., Baltimore, MD, pp. 13-16, 1998.

[43] Y. H. Fang, M. C. E. Yagoub, F. Wang, and Q. J. Zhang, “ Anew macromodeling approach for nonlinear microwave circuits based on recurrent neural networks," IEEE Trans. Microwave Theory Tech., vol. 48, pp. 2335-2344, 2000.

[44] C. Christodoulou, A. E. Zooghby, and M. Georgiopoulos, "Neural network processing for adaptive array antennas," IEEE-APS Int. Symp., Orlando, FL, pp. 2584-2587.

[45] X. Ding, B. Chattaraj, M. C. E. Yagoub, V. K. Devabhatuni, Q. J. Zhang, "EM based statistical design of microwave circuits using neural models," Int. Symp. On Microwave and Optical Technology (ISMOT 2001), Montreal, Canada, pp. 421-426, June. 2001 . 
[46] X. Ding, J. J. Xu, M. C. E. Yagoub, Q. J. Zhang, “ A new modeling approach for embedded passives exploiting state space formulation," European Microwave Conf., Milan, Italy, Sept. 23-27, 2002.

[47] Q. J. Zhang, M. C. E. Yagoub, X. Ding, D. Goulette, R. Sheffield, and H. Feyzbakhsh, "Fast and accurate modeling of embedded passives in multilayer printed circuits using neural network approach, "Elect. Components and Tech. Conf., San Diego, CA, pp. 700-703, May. 2002.

[48] G. Kothapali, "Artificial neural networks as aids in circuit design," Microelectonics $J .$, vol. 26, pp. 569-678, 1995.

[49] Q. J. Zhang, M. S. Nakhla, "Signal integrity analysis and optimization of VLSI interconnects using neural network models," IEEE Int. circuits Syst. Symp., London, England, pp. 459-462.

[50] M. D. Baker, C. D. Himmel, and G. S. May, "In-situ prediction of reactive ion etch endpoint using neural networks," IEEE Trans. Components, Packaging, and manufacturing Tech., Part A, vol. 18, pp. 478-483, 1995.

[51] M. Vai, S. Wu, B. Li, and S. Prasad, "Reverse modeling of microwave circuits with bi-directional neural network models," IEEE Trans. Microwave Theory Tech., vol. 46, pp. 1492-1494, 1998.

[52] Q. J. Zhang, K. C. Gupta and V. K. Devabhaktuni, "Artificial neural networks for RF and microwave design: from theory to practice," IEEE Trans. Microwave Theory Tech., vol. 51, pp. 1339-1350, March. 2003. 
[53] D. E. Rubelhart, G. E. Hinton, and R. J. Williams, "Learning internal presentations by error propagation, " in Parallel Distributed Processing, vol. 1, D. E. Rumelhart and J. L. McClelland, Editors, Cambridge, MA: MIT Press, pp. 318-362, 1986.

[54] D. G. Luenberger, Linear and Nonlinear Programming, Reading, MA: AddisonWesley, 1989.

[55] D. E. Goldberg, Genetic Algorithms in Search, Optimization and Machine Learning, Reading, MA: Addsion-Wdsley, 1989.

[56] S. Kirkpatrick, C. D. Gelatt, and M. P. Vecchi, "Optimization by simulated annealing," Science, vol. 220, pp. 671-680, 1983.

[57] N. Baba, Y. Mogami, M. Kohzaki, Y. Shiraishi, and Y. Yoshida, "A hybrid algorithm for finding the global minimum of error function of neural networks and its applications," IEEE Trans. Neural Networks, vol. 7, pp. 1253-1265, 1994.

[58] Q. J. Zhang, M. C. E. Yagoub, X. Ding, D. goulette, R. Sheffield, and H. Feyzbakhsh, "Fast and accurate modeling of embedded passives in multi-layer printed circuits using neural network approach," Elect. Components \& Tech. Conf., San Diego, CA, pp. 700-703, May. 2002.

[59] Q. J. Zhang, and K. C. Gupta, Neural Networks for RF and Microwave Design, Artech House, Norwood, MA, 2000.

[60] P. Burrascano, S. Fiori, and M. Mongiardo, "A review of artificial neural networks applications in microwave computer-aided design", Int. J. RF and Microwave CAE, vol. 9, pp. 158-174, 1999. 
[61] D. Saraswat, R. Achar, M. Nakhla, "A fast algorithm and practical considerations for passive macromodeling of measured/simulated data," IEEE Trans, Adv. Package., vol. 27. pp. 57-70, Feb. 2004.

[62] B. Gustavsen, and A. Semlyen, "Rational approximation of frequency domain responses by vector fitting," IEEE Trans. Power Delivery, vol. 14, pp. 1052-1061, July. 1999.

[63] NeuroModeler, v.1.5, Q. J. Zhang, Dept of Electronics, Carleton University, Ottawa, Canada.

[64] Momentum Agilent EESOF EDA, Agilent Technologies, Palo Alto, CA, USA.

[65] Sonnet v.11.54, Sonnet Software, Inc. Liverpool, NY, USA.

[66] E. Silikinson, "An N-way hybrid power divider," IRE Trans. Microwave Theory Tech., vol. MTT-8, no. 1, pp. 116-118, Jan. 1960.

[67] P. C. Goodman, "A wideband stripline matched power divider," IEEE MTT-s Int. Microwave Symp. Dig., vol 68, pp. 16-20, 1968.

[68] A. M. Abbosh, "Design of ultra-wideband three-way arbitrary power dividers," IEEE Trans. Microwave Theory Tech., vol. 56, no.1, Jan. 2008.

[69] B. L. Ooi, Palei W, M. S. Leong, "Broad-banding technique for in-phase hybrid ring equal power divider," IEEE Trans. Microwave Theory Tech., vol. 50, no.7, July. 2002.

[70] X. Ding, V. K. Devabhaktuni, B. Chattaraj, M. C. E. Yagoub, M. Doe, J. J. Xu and Q. J. Zhang, "Neural network approaches to electromagnetic based modeling of passive components and their applications to high-frequency and high-speed 
nonlinear circuit optimization," IEEE Trans. Microwave Theory Tech., vol. 52, pp. 436-449, January. 2004.

[71] Sonnet user's guide, release 11. Sonnet Software, Inc. Liverpool, NY, USA.

[72] NeuroADS, v.2.1, Q. J. Zhang, Dept of Electronics, Carleton University, Ottawa, Canada.

[73] Agilent-ADS, Agilent Technologies, Palo Alto, CA, USA.

[74] NeuroAMG, Q. J. Zhang, Dept of Electronics, Carleton University, Ottawa, Canada. 